OPEN ACCESS

Edited by:

Shao Li,

Tsinghua University, China

Reviewed by:

Mert llhan,

Yüzüncü YIl University, Turkey

Fabio Boylan,

Trinity College Dublin, Ireland

*Correspondence:

Zhigang Me

meizhigang@hnucm.edu.cn

Yang Fu

fuyangtcm@aliyun.com

Specialty section:

This article was submitted to

Ethnopharmacology,

a section of the journal

Frontiers in Pharmacology

Received: 07 February 2021

Accepted: 01 June 2021

Published: 11 June 2021

Citation:

Liu X, Fan Y, Du L, Mei Z and Fu Y (2021) In Silico and In Vivo Studies on the Mechanisms of Chinese Medicine Formula (Gegen Qinlian Decoction) in

the Treatment of Ulcerative Colitis.

Front. Pharmacol. 12:665102.

doi: 10.3389/fphar.2021.665102

\section{In Silico and In Vivo Studies on the Mechanisms of Chinese Medicine Formula (Gegen Qinlian Decoction) in the Treatment of Ulcerative Colitis}

\author{
Xiaolu Liu ${ }^{1,2}$, Yuling $\mathrm{Fan}^{2}$, Lipeng $\mathrm{Du}^{2}$, Zhigang $\mathrm{Me}^{1,2 *}$ and $\mathrm{Yang} \mathrm{Fu}^{3 *}$ \\ ${ }^{1}$ Institute of Basic Theory for Integrated Traditional Chinese and Western Medicine, College of Integrated Traditional Chinese and \\ Western Medicine, Hunan University of Chinese Medicine, Changsha, China, ${ }^{2}$ Third-Grade Pharmacological Laboratory on \\ Chinese Medicine Approved by State Administration of Traditional Chinese Medicine, Medical College of China Three Gorges \\ University, Yichang, China, ${ }^{3}$ Xiangyang Hospital of Traditional Chinese Medicine, Xiangyang, China
}

Ulcerative colitis $(\mathrm{UC})$ is a chronic inflammatory bowel disease, and Gegen Qinlian Decoction (GQD), a Chinese botanical formula, has exhibited beneficial efficacy against UC. However, the mechanisms underlying the effect of GQD still remain to be elucidated. In this study, network pharmacology approach and molecular docking in silico were applied to uncover the potential multicomponent synergetic effect and molecular mechanisms. The targets of ingredients in GQD were obtained from Traditional Chinese Medicine Systems Pharmacology Database and Analysis Platform (TCMSP) and Bioinformatics Analysis Tool for Molecular mechANism of TCM (BATMAN-TCM) database, while the UC targets were retrieved from Genecards, therapeutic target database (TTD) and Online Mendelian Inheritance in Man (OMIM) database. The topological parameters of ProteinProtein Interaction (PPI) data were used to screen the hub targets in the network. The possible mechanisms were investigated with gene ontology (GO) enrichment analysis and Kyoto Encyclopedia of Genes and Genomes (KEGG) pathway enrichment analysis. Molecular docking was used to verify the binding affinity between the active compounds and hub targets. Network pharmacology analysis successfully identified 77 candidate compounds and 56 potential targets. The targets were further mapped to 20 related pathways to construct a compound-target-pathway network and an integrated network of GQD treating UC. Among these pathways, PI3K-AKT, HIF-1, VEGF, Ras, and TNF signaling pathways may exert important effects in the treatment of UC via inflammation suppression and anti-carcinogenesis. In the animal experiment, treatment with GQD and sulfasalazine (SASP) both ameliorated inflammation in UC. The

\footnotetext{
Abbreviations: UC, ulcerative colitis; GQD, gegen qinlian decoction; TCMSP, traditional chinese medicine systems pharmacology database and analysis platform; BATMAN-TCM, bioinformatics analysis tool for molecular mechANism of TCM; TTD, therapeutic target database; OMIM, online mendelian inheritance in man; PPI, protein-protein interaction; GO, dene ontology; KEGG, kyoto encyclopedia of genes and genomes; SASP, sulfasalazine; IBD, inflammatory bowel disease; TCM, traditional chinese medicine; GG, pueraria lobata (Willd.) ohwi (Ge-Gen in Chinese); HQ, scutellaria baicalensis georgi (Huang-Qin in Chinese); HL, coptis chinensis franch (Huang-Lian in Chinese); GC, glycyrrhiza uralensis fisch (Gan-Cao in Chinese); OB, oral bioavailability; Caco-2, Caco-2 cell permeability; DL, drug-likeness; DAVID, database for annotation, visualization, and integrated discovery; DSS, dextran sulfate sodium; HPLC, high-performance liquid chromatography; TNF- $\alpha$, tumor necrosis factor- $\alpha$; IL-1 $\beta$, interleukin-1 beta; IL-6, interleukin-6; EGFR, epidermal growth factor receptor erbB1; PI3K, phosphoinositide 3-kinase; AKT, serine/threonine-protein kinase akt-1; Q-marker, quality marker.
} 
proinflammatory cytokines (TNF- $\alpha, I L-1 \beta$, and IL-6) induced by UC were significantly decreased by GQD and SASP. Moreover, the protein expression of EGFR, PI3K, and phosphorylation of AKT were reduced after GQD and SASP treatment, and there was no significance between the GQD group and SASP group. Our study systematically dissected the molecular mechanisms of GQD on the treatment of UC using network pharmacology, as well as uncovered the therapeutic effects of GQD against UC through ameliorating inflammation via downregulating EGFR/PI3K/AKT signaling pathway and the proinflammatory cytokines such as TNF- $\alpha, \mathrm{IL}-1 \beta$ and IL-6.

Keywords: gegen qinlian decoction (GQD), network pharmacology, molecular docking, inflammatory bowel disease, ulcerative colitis

\section{INTRODUCTION}

Ulcerative colitis (UC), a subcategory of inflammatory bowel disease (IBD), is an idiopathic chronic inflammatory disorder of intestinal mucosa or lamina propria (Torres, et al., 2012). The cardinal symptom of this disease can range from mild to severe with many characteristics, including abdominal pain, diarrhea, rectal urgency, and bloody stool (Head and Jurenka, 2003). In recent years, the overall incidence and prevalence of UC is reported to be increased per year (Shivashankar et al., 2017). However, the occurrence and development of UC are connected to the etiology and pathology of multiple factors in human body, such as age, sex, genetics, environmental factors and autoimmunity, which makes UC a complex disease (Ordás et al., 2012). Notably, with continuous infiltration of immune cells and repeated stimulation of epithelial cells in intestinal mucosa, a risk of tumor initiation on UC also has dramatically increased (Rubin et al., 2012). Effective treatment is still lacking in clinic, the traditional drug, such as sulfasalazine (SASP) and glucocorticoids, have severe side effects, which restrict their widespread use in clinic. Currently, the options of medication depend on the severity of disease, while 5-Aminosalicylate and corticosteroids are recommended for mild to moderate UC (Bressler et al., 2015). For the patients with moderate to severe UC, calcineurin inhibitors or biologic agent should be selected (Lv et al., 2014). Although there are optimized medical management to choose, some patients still require colectomy, which is an option for decreasing risk of neoplasia and improvement in health-related quality of life (Gallo et al., 2018). Therefore, up to now no regular method is fit for every patient, and patients may require multiple and integral treatment modalities to achieve best therapeutic effect. Fortunately, this happened to coincide with the theory of traditional Chinese medicine (TCM) based on the comprehensive and individual medicinal system.

TCM has been used clinically in Asia for more than 2000 years, and numerous Chinese botanical medicines can be adopted as an auxiliary treatment for UC (Sałaga et al., 2014). Gegen Qinlian decoction (GQD), a well-known Chinese medicinal formula, was originally invented by Zhongjing Zhang, an eminent herbalist in Eastern Han dynasty in China, and it has been clinically applied to treat diarrhea and dysentery for approximately 2000 years. According to the Chinese pharmacopoeia, GQD consists of four botanical drugs, namely Pueraria lobata (Willd.) Ohwi (Ge-Gen in Chinese, GG), Scutellaria baicalensis Georgi (Huang-Qin in Chinese, HQ), Coptis chinensis Franch (Huang-Lian in Chinese, HL), and Glycyrrhiza uralensis Fisch (Gan-Cao in Chinese, GC) at the ration of 8:3:3:2. Our previous study and clinical studies have revealed that GQD possessed amazing curative effects in the treatment of UC (Shijun, 2010; Yan et al., 2012; Fan et al., 2019). However, researches on GQD were limited to single pharmacological activity, such as alleviating the gastrointestinal function, anti-inflammatory and antibacterial properties, the overall relationships between compounds and pharmacological mechanisms of GQD have not been clarified in depth (Yu et al., 2005; Xu et al., 2015). Systems network pharmacology is a newly prominent field that combines multiple disciplines and techniques and attempts to probe potential molecular mechanisms and relationships by constructing biological network models (Huang et al., 2014; Kim et al., 2018a). At present, the network pharmacology analysis has been largely applied for several TCM formulae pharmacological research such as the Sini powder for the treatment of chronic hepatitis, the Banxia Xiexin decoction against irritable bowel syndrome, and the antidiabetic activity of GQD in the treatment of type 2 diabetes, as well as the potential mechanisms underlying the formulae effect have also been systematically illuminated (Li et al., 2014; Shu et al., 2018; Li et al., 2019). Thus, in current study, the newly network pharmacology-based approach was employed to integrate active compounds, targets and pathways prediction, and network analysis, which may provide novel insights into the therapeutic effects and molecular mechanisms of GQD. In addition, in vivo experiment was also conducted to reveal the underlying mechanisms of GQD in the treatment for UC.

\section{MATERIALS AND METHODS}

\section{Chemical Ingredients Database Construction}

All components of the four Chinese botanical drugs in GQD were retrieved from the Traditional Chinese Medicine Systems Pharmacology Database and Analysis Platform (TCMSP, http://lsp.nwu.edu.cn/) (Ru et al., 2014) and Bioinformatics 


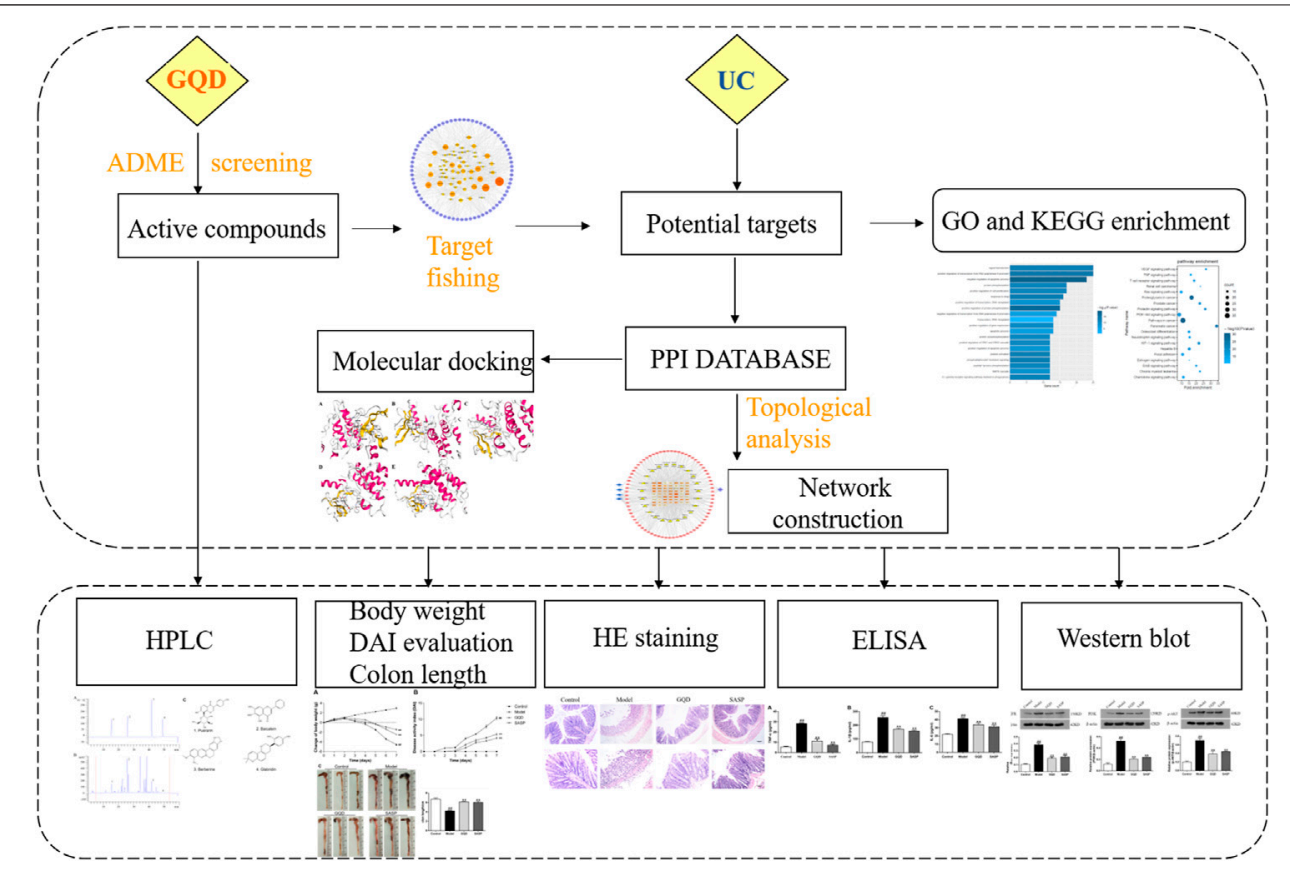

FIGURE 1 | The flowchart of network pharmacology and molecular docking-based strategy for deciphering the underlying mechanisms of GQD on the treatment of UC.

Analysis Tool for Molecular mechANism of TCM (BATMANTCM, http://bionet.ncpsb.org/batman-tcm) (Liu et al., 2016). Then we screen out the compounds which might not be matched with the criterion but were important components by a wide-scale text mining. The framework of this study was shown in Figure 1.

\section{Pharmacokinetic Selection}

To verify the pharmacokinetic characteristics of drugs, a compound screening model provided by the TCMSP data platform, including the evaluation of oral bioavailability (OB), Caco-2 cell permeability (Caco-2) and drug-likeness (DL) were employed. And the three parameters above were clarified as following. $\mathrm{OB}$ refers to the rate of an orally administered drugs of unmodified drug that delivers to circulatory system, which is considered predictive of bioactive molecule indicators as therapeutic agents (Xu et al., 2012; Chow, 2014). According to the recommended drug screening criteria, the compounds of which the threshold of $\mathrm{OB} \geq 30 \%$ in this research could be qualified as a candidate ingredient. Caco-2 is another important parameter generally used as a model to predict the intestinal drug absorption in pre-clinical investigations (Artursson and Karlsson, 1991). And the application of this model employed in screening potential botanical-drug interactions is gaining popularity (Awortwe et al., 2014). In this study, to make sure the putative ingredients of GQD have high permeability via the intestinal membrane enterocytes, we increased the threshold values to Caco- $2 \geq 0.4$. DL is a qualitative concept used in drug design for an estimate on how "drug-like" a prospective compound is, which helps to optimize pharmacokinetic and pharmaceutical properties, such as solubility and chemical stability (Tao et al., 2013). The "drug-like" level of the compounds is 0.18 , which is used as a selection criterion to filter out molecules with undesirable properties in traditional Chinese botanical drugs (Tian et al., 2015). Consequently, ingredients in database of which the parameters' information meets the above screening criteria were opted to the next step.

\section{Potential Targets Fishing}

The protein targets of the candidate substances in GQD were retrieved from The Swiss Target Prediction database (http://www. swisstargetprediction.ch/), which can be mined automatically to retrieve specific information for a large number of molecules (Gfeller et al., 2014). In Swiss Target Prediction database, query molecules can be import either as SMILES structure format or drawn in $2 \mathrm{D}$. And the molecular structure information of candidate compounds was derived from PubChem (https://pubchem.ncbi. nlm.nih.gov/), which is a pivotal chemistry resource served as an archive of chemical substance description and structure standardization (Kim et al., 2018b). Then, the target name was mapped to the disease target predictive database to find the disease associated with the protein and retrieved the diseases about UC. UCrelated human genes were collected from three resources, which consist of Genecards (http://www.genecards.org), therapeutic target database (TTD) (http://db.idrblab.net/ttd/) and Online Mendelian Inheritance in Man (OMIM) database (http://omim.org/).

\section{Protein-Protein Interaction}

The protein-protein interaction (PPI) of each target were generated from STRING database (http://string-db.org/, ver. 
11) with the species limited to "Homo sapiens" and a confidence score $>0.7$ (Szklarczyk et al., 2019). Because of the complexity of the original network generated in STRING database, we imported the PPI data into the network visualization software Cytoscape (version 3.7.2) to reconstruct the network for better visualization, and screen out the hub targets.

\section{Gene Ontology and Kyoto Encyclopedia of Genes and Genomes Pathway Enrichment Analysis}

To systematically explore the concerned biological processes of GQD as a therapy against UC, we performed GO and KEGG enrichment analysis by the functional annotation tool DAVID (database for annotation, visualization, and integrated discovery; http://david.abcc.ncifcrf.gov). GO terms and KEGG pathways with $p$ value less than 0.05 according to Fisher's exact test were considered statistically significant and subjected to further analyses.

\section{Network Construction}

To comprehensive understand the molecular mechanisms of GQD to UC, the networks including compound-targetpathway network were constructed using Cytoscape version 3.7.2. The construction of the network was based on the binding of active ingredients to the correlative targets and the signaling pathways, which uncover the underlying interactions between botanical drugs and the disease. In this graphical network, nodes represent the compounds, targets, or signaling pathways by different shapes, and the interactions between them were signified by edges. The degree of a node was marked by different color or size, which was defined as the number of edges relate to it. Moreover, the topological properties were analyzed by the plugin Network Analyzer of Cytoscape (Shannon et al., 2003).

\section{Molecular Docking}

Molecular docking of the critical active compounds with hub targets was performed by CB-Dock (http://cao.labshare.cn/cbdock/), a web used for predicting the binding sites and affinity with a popular docking program, Autodock Viana (Liu et al., 2020). CB-Dock is a new blind docking tool, which focus on enhancing the docking accuracy. PDB formats of the proteins and a ligand file in the MOL2 or SDF were input to CB-dock to generate a set of points to represent the solvent-accessible surface, and the cavities were ranked according to their sizes. Then a docking box for the following computation was customized, and irrelevant poses was excluded as many as possible. Then we predicted the binding sites, calculated the centers and sizes with a novel curvature-based cavity detection approach, and performed ligand docking with Autodock Vina, and obtained the binding activities and the Vina score, which represents the binding affinity. The more negative the Vina score is, the more stable the ligand-receptor binding (Liu et al., 2020). Ball and stick and cartoon chain represented the ligand and protein, respectively.

\section{Animals}

A total of 60 male C57BL/6 mice weighing about 18-22 g were purchased from the Laboratory Animal Center of China Tree
Gorges University (Yichang, China). All mice were randomly assigned to four groups ( $n=15 /$ group): control group, model group, GQD group and SASP group. All animals were maintained under a $12 \mathrm{~h}$ light/dark cycle environment. The room temperature was controlled at $(22 \pm 1)^{\circ} \mathrm{C}$, with $(60 \pm 5) \%$ humidity, and adequate food and water were provided. The animal experiments were approved by the Laboratory Animal Ethical Committee of Three Gorges University, China (No. 20180904C, approved at 2018-9-11).

\section{Drugs and Reagents}

The botanical formula GQD is comprised of four medicinal botanical drugs: Pueraria lobata (Willd.) Ohwi (Batch No: 202011027, Hubei Tianji Pharmaceutical Co. Ltd.), Scutellaria baicalensis Georgi (Batch No: 20201103, Hubei Tianji Pharmaceutical Co. Ltd.), Coptis chinensis Franch (Batch No: 202011019, Hubei Tianji Pharmaceutical Co. Ltd.), and Glycyrrhiza uralensis Fisch (Batch No: 202012018, Hubei Tianji Pharmaceutical Co. Ltd.). All the botanical drugs were purchased from Yichang Hospital of Traditional Chinese Medicine, Hubei province, China. Dextran sulfate sodium (DSS; molecular weight: $36-50 \mathrm{kDa}$ ) was obtained from MP Biomedicals (Santa Ana, CA, United States). EGFR antibody, and PI3K antibody were obtained from Proteintech Group (Chicago, United States); $p$-AKT antibody was purchased from Affinity Biologicals (Yarker, Canada), and $\beta$-actin antibody was obtained from Boster Biological Technology (Wuhan, China).

\section{Preparation of Gegen Qinlian Decoction Extract}

For the extraction of GQD, Pueraria lobata (Willd.) Ohwi (24 g), Scutellaria baicalensis Georgi (9 g), Coptis chinensis Franch (9 g), and Glycyrrhiza uralensis Fisch (6 g) were soaked in 75\% ethanol at eightfold volume overnight and then boiled for $1.5 \mathrm{~h}$, the first decoction was thus obtained. Then the residue was boiled a second time with an addition of sixfold volume of $75 \%$ ethanol for $1 \mathrm{~h}$ to obtain the second decoction. Finally, the first and second decoction were mixed, filtered through gauze, and evaporated to dryness under reduced pressure, and then the extractum was freeze drying to powder.

\section{Component Analysis of Gegen Qinlian Decoction With High-Performance Liquid Chromatography}

The analytical standards were purchased from Push Biotechnology Co., Ltd. (Chengdu, China), and the analysis of GQD was performed on Agilent 1,260 Infinity II HPLC system (Agilent Technologies, Santa Clara, CA, United States) equipped with a Diode Array Detector (DAD). A reversed phase Welch Ultimate XB C18 $(25 \times 4.6 \mathrm{~mm}, 5 \mu \mathrm{m})$ was used and kept at $30^{\circ} \mathrm{C}$. The mobile phase consisted of aqueous solution of phosphoric acid and methanol with a gradient elution at a flow rate of $1.0 \mathrm{ml} /$ min. And the detection wavelength was set at 250, 350, 280, and $250 \mathrm{~nm}$. 


\section{Treatment Protocol for Dextran Sulfate Sodium-Induced Colitic Mice}

Mice were randomly allocated to four groups ( $n=15$ /group): control group, model group, GQD group, and SASP group. All mice except the control group were induced by administration of $3 \%$ DSS dissolved in drinking water continuously for 7 days. And the mice in normal group received distilled water without DSS throughout the entire experimental period. According to clinical administration dose, the mice in GQD group and SASP group were administered with GQD enema ( $0.68 \mathrm{~g} / \mathrm{kg}$ per day) or SASP enema $(0.5 \mathrm{~g} / \mathrm{kg}$ per day), respectively. Meanwhile, the mice in control group and model group were administrated with saline by enemata. The animals were monitored once a day for body weight, stool consistency, and presence of blood in the feces and the anus. At day 7, mice were anesthetized and sacrificed by cervical dislocation, and the colon samples were collected immediately. Then the colon length was measured and stored for subsequently experiments.

\section{Evaluation of Disease Activity and Colon Length}

To assess the severity of colitis, the disease activity index (DAI) was calculated as the average of body weight loss score, diarrhea score, and fecal blood score. DAI evaluation was calculated under the guidance of previously established scoring system (Yan et al., 2018b; Hassan and Hassan, 2018), and the scoring criteria was based on the following parameters: 1$)$ body weight loss ( 0 , no loss; 1, 1-5\% loss; 2, 5-10\% loss; 3, 10-20\% loss; 4, over 20\% loss); 2) diarrhea ( 0 , normal; 2 , loose stools; 4 , watery diarrhea); and 3) hematochezia (0, no bleeding; 2, slight bleeding; 4, gross bleeding). Body weight gain was expressed as the difference from the initial body weight. Colon length was regarded as an indirect marker of inflammation, thereby, the length of colons from the ileocecal junction to the anal verge was measured.

\section{Histopathological Analysis}

Colon tissues were collected and fixed in 4\% paraformaldehyde overnight, then dehydrated at gradient concentrations of ethanol, and embedded in paraffin. Tissues were sectioned at $4 \mu \mathrm{m}$ thickness with a paraffin and stained with hematoxylin and eosin (HE). The images of the tissues were obtained using Image-Pro Plus 5.0 system.

\section{Enzyme-Linked Immunosorbent Assay}

To test the anti-inflammatory effects of GQD on ulcerative colitis, colon homogenate supernatants of all the experimental groups were collected, and the expression of TNF- $\alpha$, IL- $1 \beta$, and IL- 6 was measured by ELISA kits (USCN Cloud-Clone Corp, Wuhan, China) according to the manufacturer's instructions.

\section{Western Blotting Analysis}

The total protein of colon tissue was extracted using RIPA lysis buffer with the protease inhibitor cocktail (Beyotime Biotechnology, China), and protein concentration was measured using the BCA protein assay kit (Beyotime
Biotechnology) according to the manufacturer's instructions. Equivalent amounts of protein $(20 \mu \mathrm{g})$ were separated by SDSPAGE and then transferred onto $0.45 \mu \mathrm{m}$ PVDF membranes (Millipore, Billeria, MA, United States). The membranes were then blocked in 5\% nonfat milk in TBST buffer for $1 \mathrm{~h}$ at room temperature, followed by incubation with primary antibodies at $4^{\circ} \mathrm{C}$ overnight. Then membranes were washed with TBST and incubated with secondary antibody for $1 \mathrm{~h}$ at room temperature, proteins were detected using ECL reagent (Applygen, China). The antibodies used were as follows: EGFR (1:2,000), PI3K (1:1,000), p-AKT $(1: 1,000)$, and $\beta$-actin (1:500).

\section{Statistical Analysis}

All the experimental data were analyzed using SPSS 20.0 statistical software (IBM Corp., Armonk, NY, United States). GraphPad Prism 6.0 software (GraphPad Software Inc., San Diego, CA, United States) was used for the calculations. All results are expressed as means \pm standard error of the mean (SEM). Multigroup comparisons were performed using one-way analysis of variance (ANOVA) and post hoc Statistical least significant difference (LSD) test. $p$ value $<0.05$ was considered statistically significant.

\section{RESULTS}

\section{Identification of Candidate Compounds in Gegen Qinlian Decoction}

After the absorption, distribution, metabolism, elimination (ADME) parameters screening ( $\mathrm{OB} \geq 30 \%$, DL $\geq 0.18$, Caco- 2 $\geq 0.4$ ), 75 potential components of the four botanical medicines were collected from database and relate literatures. Among them, 3 ingredients belong to GG, 9 belong to HL, 20 belong to HQ, and 47 belong to GC. It is worth mentioning that formononetin $(\mathrm{OB}=$ $69.67 \%$, Caco-2 $=0.78, \mathrm{DL}=0.21$ ) as an important component existed in both GG and GC. Additionally, beta-sitosterol (OB = $39.91 \%$, Caco-2 = 1.32, DL = 0.75), exhibited good ADME properties, can be collected from GG and HQ. Both coptisine $(\mathrm{OB}=30.67 \%$, Caco- $2=1.21, \mathrm{DL}=0.86)$ and epiberberine $(\mathrm{OB}=$ $43.09 \%$, Caco- $2=1.17, \mathrm{DL}=0.78$ ) are common ingredients found in HQ and HL. Additionally, two compounds, puerarin and daidzein, did not meet the three criteria were taken into consideration as their obvious bioactivities (Qi et al., 2004). Consequently, after removing redundancy, a total of 77 compounds were identified as candidate compounds for further analysis. All the compounds identified from GQD and their concreted predicted OB, DL, and Caco- 2 values are showed in Table 1.

\section{Targets Identification of Gegen Qinlian Decoction on Ulcerative Colitis}

Based on The Swiss Target Prediction database, a total of 740 targets related to the 77 compounds were obtained in GQD. In order to explore the relevance and underlying mechanism of these targets specific to GQD in the development of UC, A total of 3785 UC-related genes were retrieved from the GeneCards, 
TABLE 1 | Information for the candidate bioactive compounds of GQD.

\begin{tabular}{|c|c|c|c|c|c|}
\hline ID & Chemical & OB (\%) & Caco-2 & DL & Herb \\
\hline C01 & Puerarin & 24.03 & -1.15 & 0.69 & GG \\
\hline C02 & Formononetin & 69.67 & 0.78 & 0.21 & GG GC \\
\hline C03 & Daidzein & 19.44 & 0.59 & 0.19 & $G G$ \\
\hline C04 & Beta-sitosterol & 36.91 & 1.32 & 0.75 & GG HQ \\
\hline C05 & $3^{\prime}$-methoxydaidzein & 48.57 & 0.56 & 0.24 & GG \\
\hline C06 & Coptisine & 30.67 & 1.21 & 0.86 & HQ HL \\
\hline $\mathrm{CO7}$ & Epiberberine & 43.09 & 1.17 & 0.78 & HQ HL \\
\hline C08 & Berberine & 36.86 & 1.24 & 0.78 & $\mathrm{HL}$ \\
\hline C09 & Berberrubine & 35.74 & 1.07 & 0.73 & $\mathrm{HL}$ \\
\hline C10 & (R)-canadine & 55.37 & 1.04 & 0.77 & $\mathrm{HL}$ \\
\hline C11 & Berlambine & 36.68 & 0.97 & 0.82 & $\mathrm{HL}$ \\
\hline C12 & Palmatine & 64.60 & 1.33 & 0.65 & $\mathrm{HL}$ \\
\hline C13 & Worenine & 45.83 & 1.22 & 0.87 & $\mathrm{HL}$ \\
\hline C14 & Moupinamide & 86.71 & 0.55 & 0.26 & $\mathrm{HI}$ \\
\hline C15 & $5,2^{\prime}, 6^{\prime}$-trihydroxy-7,8-dimethosyflavone & 45.05 & 0.48 & 0.33 & $H Q$ \\
\hline C16 & 5,2'-dihydroxy-6,7,8-trimethoxyflavone & 31.71 & 0.93 & 0.35 & $H Q$ \\
\hline C17 & 5,7,4'-trihydroxy-6-methoxyflavanone & 36.63 & 0.43 & 0.27 & $H Q$ \\
\hline C18 & Acacetin & 34.97 & 0.67 & 0.24 & $H Q$ \\
\hline C19 & Baicalein & 33.52 & 0.63 & 0.21 & $H Q$ \\
\hline C20 & Dihydrobaicalin & 40.04 & 0.56 & 0.21 & $H Q$ \\
\hline $\mathrm{C} 21$ & Dihydrooroxylin & 66.06 & 0.67 & 0.23 & $H Q$ \\
\hline $\mathrm{C} 22$ & Moslosooflavone & 44.09 & 1.01 & 0.25 & $\mathrm{HQ}$ \\
\hline C23 & Norwogonin & 39.40 & 0.60 & 0.21 & $H Q$ \\
\hline $\mathrm{C} 24$ & Oroxylin a & 41.37 & 0.76 & 0.23 & $\mathrm{HQ}$ \\
\hline C25 & Panicolin & 76.26 & 0.84 & 0.29 & $H Q$ \\
\hline C26 & Rivularin & 37.94 & 0.65 & 0.37 & $\mathrm{HQ}$ \\
\hline $\mathrm{C} 27$ & Salvigenin & 49.07 & 0.86 & 0.33 & $H Q$ \\
\hline C28 & Skullcapflavone II & 69.51 & 0.68 & 0.44 & $\mathrm{HQ}$ \\
\hline C29 & Stigmasterol & 43.83 & 1.44 & 0.76 & $H Q$ \\
\hline С30 & Supraene & 33.55 & 2.08 & 0.42 & $H Q$ \\
\hline C31 & Wogonin & 30.68 & 0.79 & 0.23 & $H Q$ \\
\hline C32 & 1-Methoxyphaseollidin & 69.98 & 1.01 & 0.64 & GC \\
\hline C33 & $3^{\prime}$-hydroxy-4'-O-Methylglabrindin & 43.71 & 1.00 & 0.57 & GC \\
\hline C34 & $3^{\prime}$-methoxyglabridin & 46.16 & 0.94 & 0.57 & GC \\
\hline C35 & 7-Acetoxy-2-methylisoflavone & 38.92 & 0.74 & 0.26 & GC \\
\hline C36 & 7-Methoxy-2-methylisoflavone & 42.56 & 1.16 & 0.20 & GC \\
\hline C37 & Calycosin & 47.75 & 0.52 & 0.24 & GC \\
\hline C38 & DFV & 32.76 & 0.51 & 0.18 & GC \\
\hline C39 & Eurycaprin A & 43.28 & 0.43 & 0.37 & GC \\
\hline C40 & Gancaonin A & 51.08 & 0.80 & 0.40 & GC \\
\hline C41 & Gancaonin B & 48.79 & 0.58 & 0.45 & GC \\
\hline C42 & Gancaonin G & 60.44 & 0.78 & 0.39 & GC \\
\hline C43 & Glabranin & 52.90 & 0.97 & 0.31 & GC \\
\hline C44 & Glabrene & 46.27 & 0.99 & 0.44 & GC \\
\hline C45 & Glabridin & 53.25 & 0.97 & 0.47 & GC \\
\hline C46 & Glabrone & 52.51 & 0.59 & 0.50 & GC \\
\hline C47 & Glepidotin A & 44.72 & 0.79 & 0.35 & GC \\
\hline C48 & Glyasperin B & 65.22 & 0.47 & 0.44 & $\mathrm{GC}$ \\
\hline C49 & Glyasperin C & 45.56 & 0.71 & 0.40 & GC \\
\hline C50 & Glyasperin F & 75.84 & 0.43 & 0.54 & GC \\
\hline C51 & Glycyrin & 52.61 & 0.59 & 0.47 & GC \\
\hline C52 & Glycyrol & 90.78 & 0.71 & 0.67 & GC \\
\hline C53 & Glypallichalcone & 61.60 & 0.76 & 0.19 & GC \\
\hline C54 & $\mathrm{HMO}$ & 38.37 & 0.79 & 0.21 & GC \\
\hline C55 & Inermine & 75.18 & 0.89 & 0.54 & GC \\
\hline C56 & Inflacoumarin A & 39.71 & 0.73 & 0.33 & $\mathrm{GC}$ \\
\hline C57 & Isoglycyrol & 44.70 & 0.91 & 0.84 & GC \\
\hline C58 & Isolicoflavonol & 45.17 & 0.54 & 0.42 & GC \\
\hline C59 & Isotrifoliol & 31.94 & 0.53 & 0.42 & $\mathrm{GC}$ \\
\hline C60 & Jaranol & 50.83 & 0.61 & 0.29 & GC \\
\hline C61 & Kanzonols W & 50.48 & 0.63 & 0.52 & GC \\
\hline C62 & Licoagrocarpin & 58.81 & 1.23 & 0.58 & GC \\
\hline C63 & Licoagroisoflavone & 57.28 & 0.71 & 0.49 & $\mathrm{GC}$ \\
\hline C64 & Licochalcone A & 40.79 & 0.82 & 0.29 & GC \\
\hline
\end{tabular}


TABLE 1 | (Continued) Information for the candidate bioactive compounds of GQD.

\begin{tabular}{|c|c|c|c|c|c|}
\hline ID & Chemical & OB (\%) & Caco-2 & DL & Herb \\
\hline C65 & Licochalcone B & 76.76 & 0.47 & 0.19 & GC \\
\hline C66 & Licochalcone G & 49.25 & 0.64 & 0.32 & GC \\
\hline C67 & Licoisoflavone B & 38.93 & 0.46 & 0.55 & GC \\
\hline C68 & Licoricone & 63.58 & 0.53 & 0.47 & GC \\
\hline C69 & Lupiwighteone & 51.64 & 0.68 & 0.37 & GC \\
\hline $\mathrm{C} 70$ & Mairin & 55.38 & 0.73 & 0.78 & GC \\
\hline $\mathrm{C} 71$ & Medicarpin & 49.22 & 1.00 & 0.34 & GC \\
\hline $\mathrm{C} 72$ & Odoratin & 49.95 & 0.42 & 0.30 & GC \\
\hline C73 & Phaseol & 78.77 & 0.76 & 0.58 & GC \\
\hline $\mathrm{C} 74$ & Phaseolinisoflavan & 32.01 & 1.01 & 0.45 & $\mathrm{GC}$ \\
\hline C75 & Semilicoisoflavone B & 48.78 & 0.45 & 0.55 & GC \\
\hline C76 & Shinpterocarpin & 80.30 & 1.10 & 0.73 & GC \\
\hline $\mathrm{C} 77$ & Vestitol & 74.66 & 0.86 & 0.21 & GC \\
\hline
\end{tabular}

$O B$, oral bioavailability; Caco-2, Caco-2 cell permeability; DL, drug-likeness.

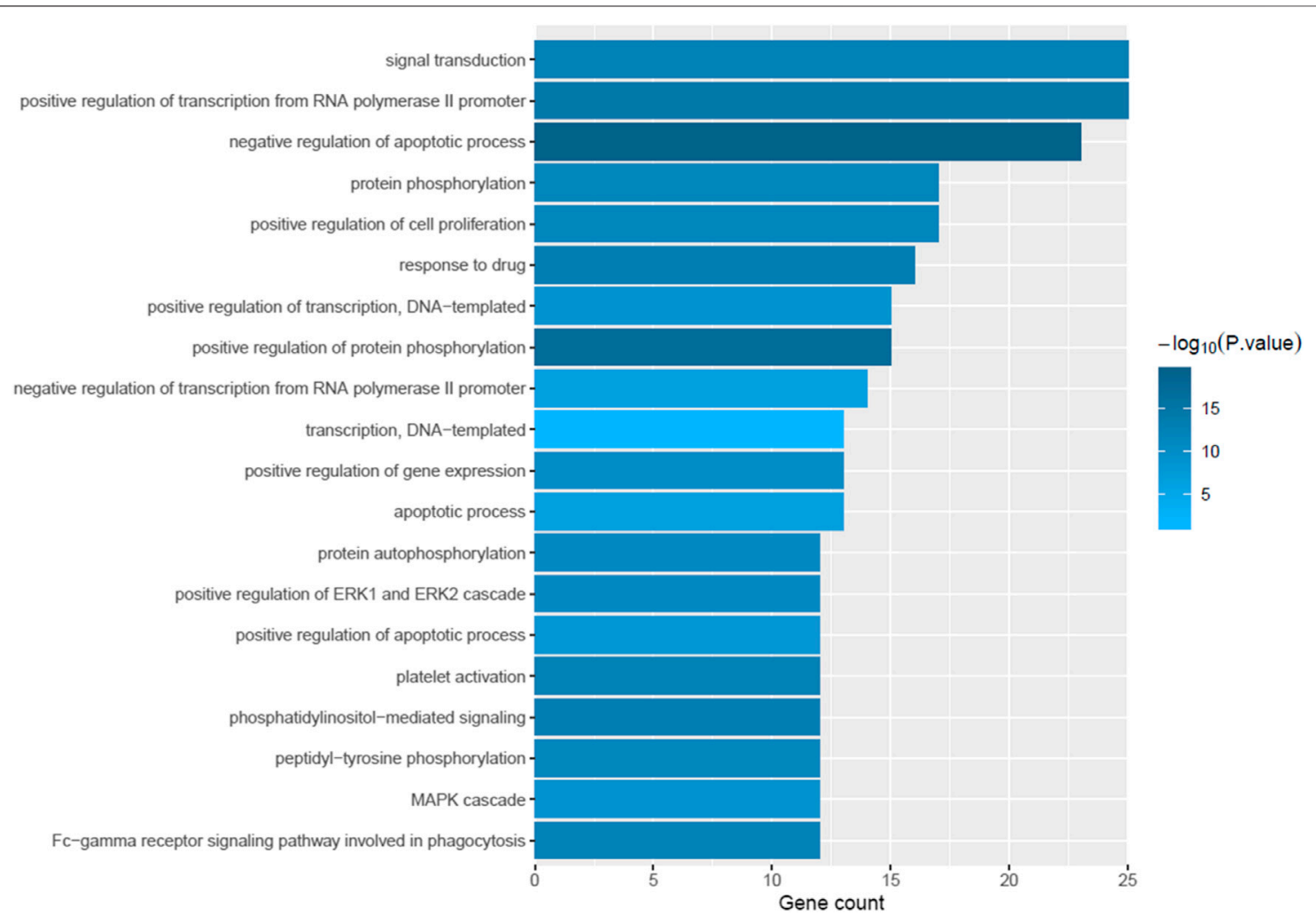

FIGURE 2| The results of gene ontology (GO) biological process analysis. The X-axis represents gene count, while the $Y$-axis represents the categories of biological process ( $p$-value $\leq 0.05)$.

OMIM, and TTD databases by employing a keyword-based search. After eliminating the overlaps, 287 targets were shared between 740 compound-related targets and 3785 UC-related targets. PPI network of these 287 targets were established in STRING database and visualized by Cytoscape software. There were 287 nodes and 4,490 edges in this interaction network. According to previous study, a node would be defined as a hub target when the degree was more than twofold the median degree of all nodes in the network (Li et al., 2007). Eventually, 59 major hub targets were considered to be the effective therapeutic targets of UC. Topological analysis indicated that serine/threonineprotein kinase AKT (AKT1, degree = 165), vascular endothelial growth factor A (VEGFA, degree = 151), epidermal growth factor receptor erbB1 (EGFR, degree $=141)$, MAP kinase ERK1 (MAPK3, degree = 141), TNF-alpha (TNF, degree $=136)$, signal transducer and activator of transcription3 


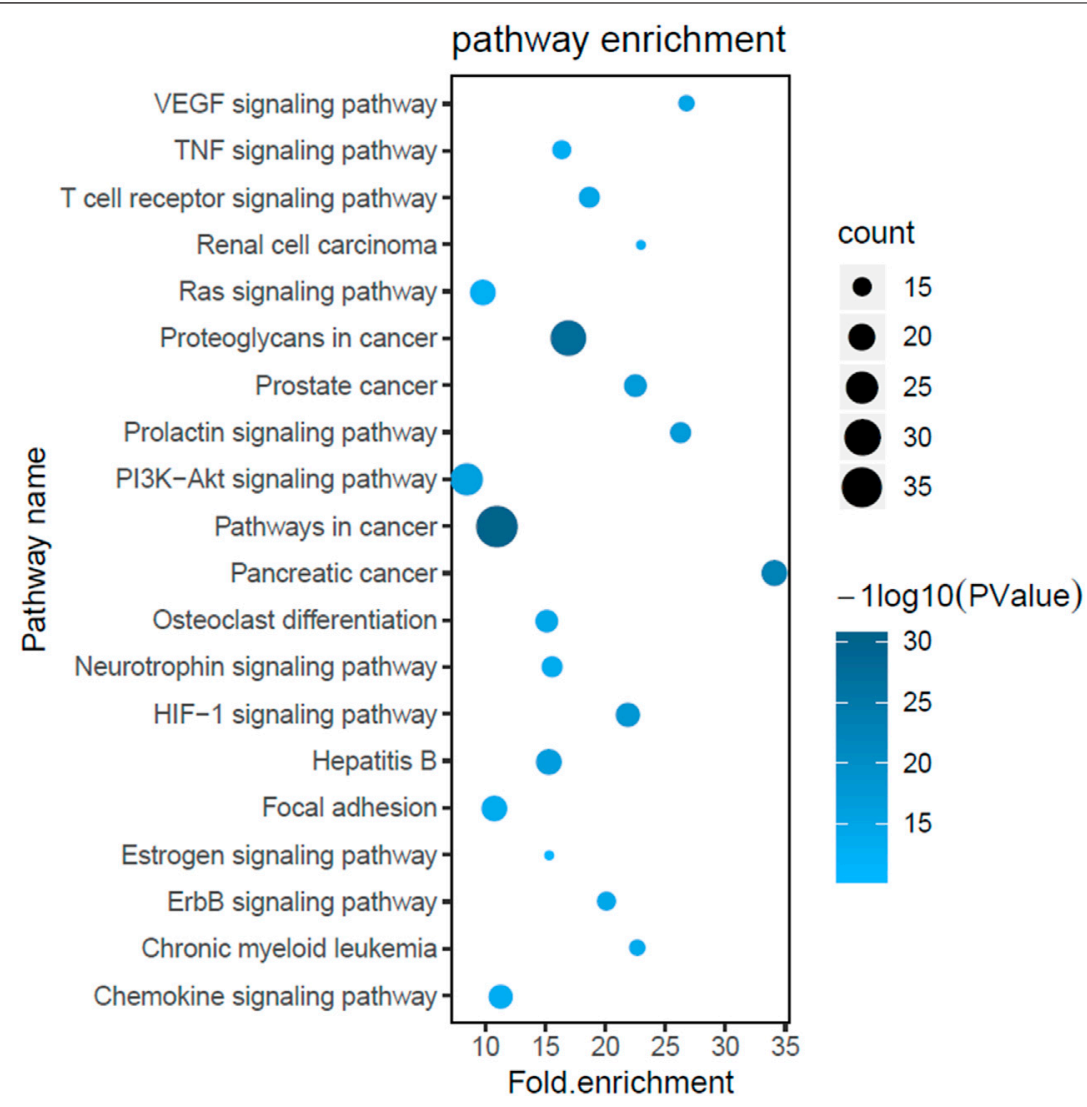

FIGURE 3 | Kyoto Encyclopedia of Genes and Genomes (KEGG) enrichment analysis. The X-axis represents the enrichment rate of these genes in total genes, while the $Y$-axis represents the enrichment pathways of the target genes ( $p$-value $\leq 0.05)$. The depth of the color represents the size of the value, and the size of circle represents the enrichment counts of these pathways.

(STAT3, degree $=128)$, tyrosine-protein kinase SRC (SRC, degree $=126)$, cyclooxygenase-2 $($ PTGS2, degree $=113)$ were the top hub targets based on degree centrality.

\section{Gene Ontology and Pathway Enrichment Analysis}

To explore the biological processes of the 59 putative targets of GQD on UC, GO and pathway enrichment analysis was further performed. As shown in Figure 2, the top 20 most significantly enriched GO terms were exhibited with their $p$-value and gene count. These targets were obviously enriched in negative regulation of apoptotic process, protein phosphorylation, cell proliferation, MAPK cascade and inflammatory response, which have a strongly correlation with the pathogenesis of UC. These results indicated the multiple synergies of GQD on biological processes. To further reveal the potential mechanisms of the therapeutic effects of GQD, we conducted KEGG pathway enrichment analysis on 59 targets, and screened out 20 pathways based on the threshold of $p$-value $<0.05$. The top 20 significantly enriched pathways contained pathways in cancer, HIF-1 signaling pathway, PI3K-AKT signaling pathway, TNF signaling pathway, VEGF signaling pathway and Ras signaling pathway (Figure 3).

\section{Compound-Target-Pathway Network Analysis}

To further elucidate the underlying pharmacological mechanisms of the candidate compounds for the treatment of GQD, the compound-target network and compound-target-pathway network were constructed based on the protein targets and the corresponding pathway enrichment analysis. As shown in Figure 4, the compound-target network was composed of 132 nodes and 684 edges, and the average degree value of hub targets was 9.03. The epiberberine $(\mathrm{C} 07$, degree $=26)$ from HQ and HL, acacetin $(\mathrm{C} 18$, degree $=18)$ and the baicalein $(\mathrm{C} 19$, degree $=16)$ from HQ, berberine (C08, degree = 13) from HL, wogonin (C31, degree $=13)$ from $\mathrm{HQ}$, glabridin $(\mathrm{C} 45$, degree $=12)$ from GC were identified as relatively high-degree active compounds, which indicated that the six compounds play more important roles in the pathological processes of ulcerative colitis. According to previous studies, puerarin, one of the most important constituents in GG, has been widely used in the treatment of UC by inhibiting inflammatory response (Jeon et al., 2020). Therefore, we hypothesize that epiberberine, acacetin, baicalein, berberine, wogonin, glabridin and puerarin may account for the essential therapeutic effects of GQD on UC. Then we selected four representative compounds of each of the four botanical drugs for molecular docking and HPLC analysis. 


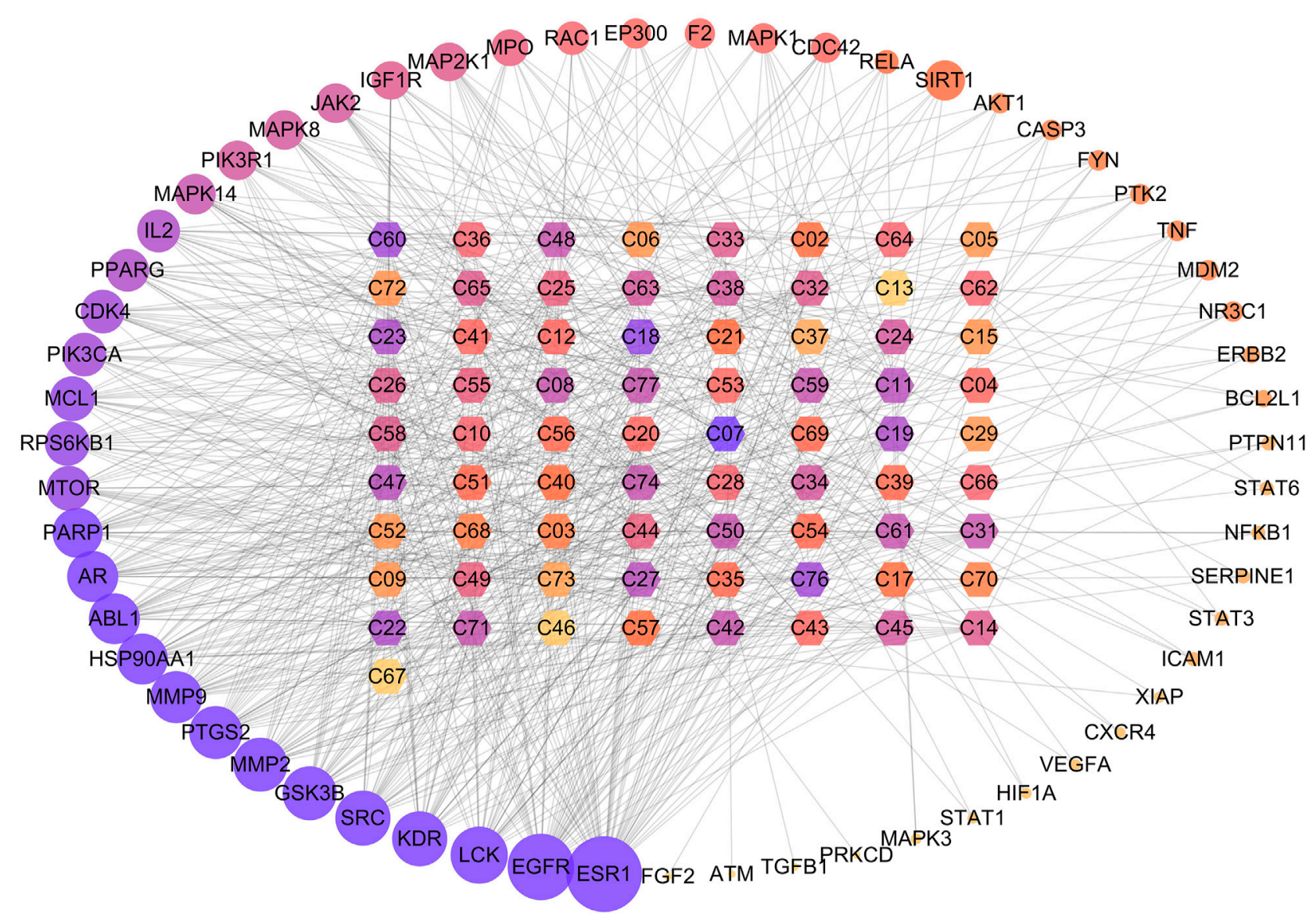

FIGURE 4 | The interaction network between compounds and hub targets. The hexagon represents the herbal compounds, the circles stand for the potential targets, and the edges represent the interactions between them. The depth of color and the size of circle are proportional to their degree value. The ID of the compounds was elaborated in Table $\mathbf{1 .}$

Among these compounds, puerarin from GG, baicalein from HQ, berberine from HL, and glabridin from GC were taken into consideration. In the meantime, the 59 hubs with higher degree scores, such as estrogen receptor alpha (ESR1, degree = 47 ), epidermal growth factor receptor erbB1 (EGFR, degree $=38$ ), tyrosine-protein kinase SRC (SRC, degree $=27$ ), matrix metalloproteinase $2(\mathrm{MMP} 2$, degree $=26)$, glycogen synthase kinase- 3 beta (GSK3B, degree $=26)$, cyclooxygenase-2 (PTGS2, degree $=25)$ possessed highly connection with compounds and pathways (Figure 5). Detailed information about the hub targets is provided in Table 2. Furthermore, an investigation at the relationship between 59 targets and 20 remarkably enriched pathways was conducted based on the compound-targetpathway network, and the average degree value of hub targets per pathway was 18.39 . These pathways probably attributed to the therapeutic effect of GQD on UC.

\section{Molecular Docking}

To further verify the results of network pharmacology screening, the four crucial active compounds in GQD were docked with the hub target EGFR via CB-dock. It is generally believed that the vina score indicates the binding affinity between the ligand and the receptor, and the more negative the vina score is, the more stable the compounds bind to the hub target. As shown in Figure 6, the crucial active components in GQD, which consist of puerarin, baicalein, berberine and glabridin, all possessed good binding activities to EGFR. And puerarin exhibited the best binding affinity with EGFR, which was higher than the positive control drug SASP.

\section{High-Performance Liquid Chromatography Analysis of Gegen Qinlian Decoction}

As shown in Figure 7, HPLC analysis indicated the retention time of standards puerarin (15.429 $\mathrm{min})$, baicalein $(25.764 \mathrm{~min})$, berberine (41.765 $\mathrm{min})$ and glabridin (49.096 min), respectively, in Figure 6A. Moreover, comparison with mixed check samples were performed, and external method was used to calculate the concentrations. The contents of puerarin, baicalein, berberine and glabridin were $1,290.93 \mu \mathrm{g} / \mathrm{g}(2.52 \%), 6,616.57 \mu \mathrm{g} / \mathrm{g}$ $(11.35 \%), \quad 1,129.38 \mu \mathrm{g} / \mathrm{g} \quad(2.8 \%)$ and $5.49 \mu \mathrm{g} / \mathrm{g} \quad(0.57 \%)$, respectively. The results showed that GQD contained the four active components. In addition, there were many peaks from 


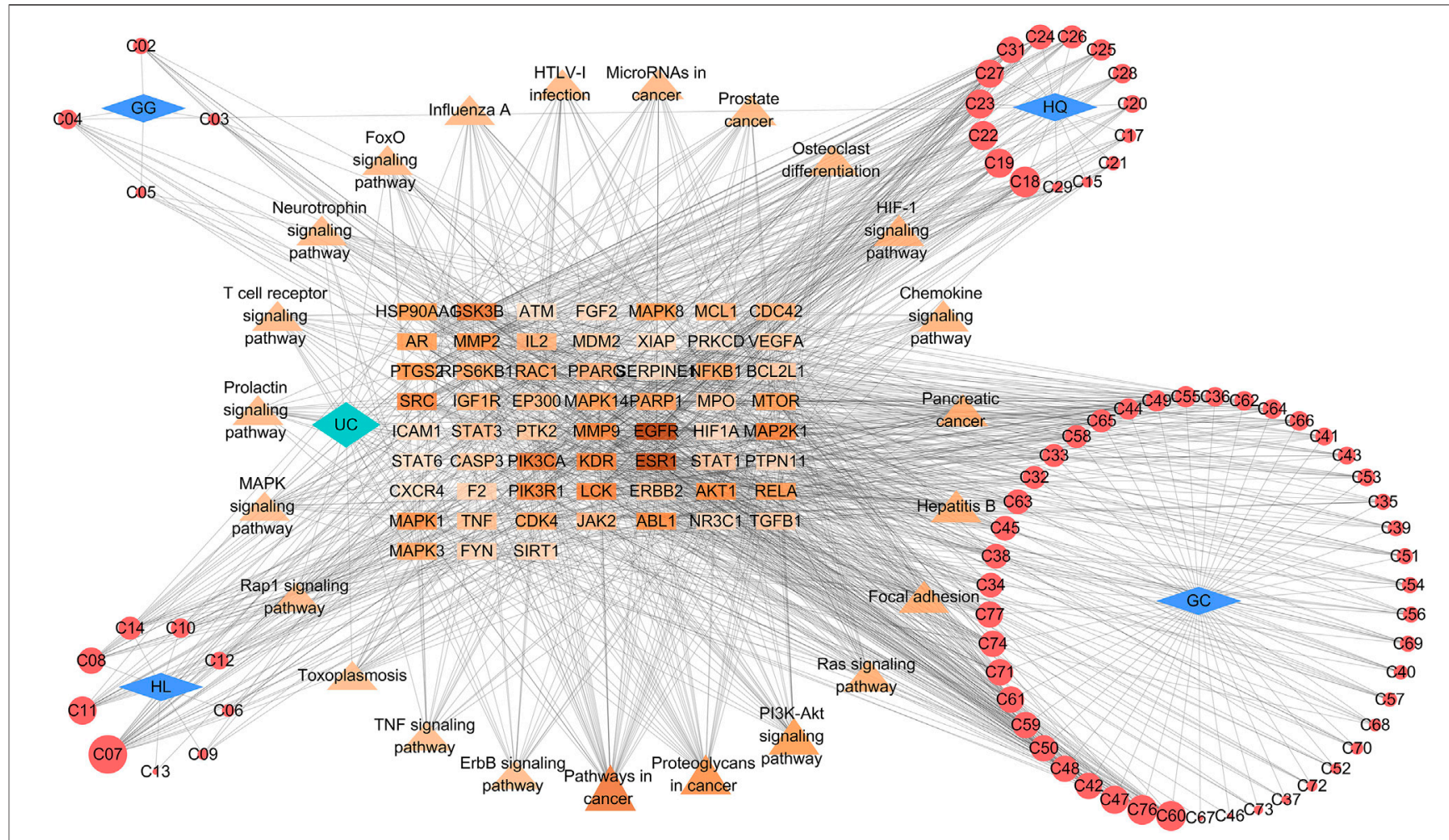

FIGURE 5 | Compound-target-pathway network. The rectangle, triangle and circle represent the potential targets, major pathways and botanical compounds, respectively. The rhombus represents the four botanical drugs in GQD. Edges represent the interaction between them. For the potential targets and pathways, the change in color depth reflect the degree value. For the botanical compounds, the circle size is proportional to their degree value. GG, Pueraria lobata (Willd.) Ohwi; HQ, Scutellaria baicalensis Georgi; HL, Coptis chinensis Franch; GC, Glycyrrhiza uralensis Fisch.

more concentrated compounds that were not analyzed quantitatively or qualitatively in the chromatograms of GQD. It has been demonstrated that the glycyrrhizic acid and baicalin, which belong to triterpene saponins and $O$-glycosides, respectively, possess protective effects against non-alcoholic fatty liver disease and diabetes (Wang et al., 2016; Yan et al., 2018a; Qiao et al., 2018). Therefore, the high concentrated compounds in HPLC chromatogram may be the compounds mentioned above.

\section{Gegen Qinlian Decoction Ameliorated Dextran Sulfate Sodium-Induced Ulcerative Colitis in Mice}

To explore the protective effects of GQD on UC, the mouse model of acute ulcerative colitis was established by administrating mice with $5 \%$ DSS for 7 days. SASP was served as a positive control in this study. The therapeutic effect of GQD on UC was assessed preliminary by body weight loss, colon length and DAI score. On the 3rd day of DSS induction, body weight of model group was markedly decreased compared to sham group, with a continuous daily decrease thereafter. And administration of GQD and SASP significantly blunted the body weight loss induced by DSS compared with model group $(p<0.01$, Figure 8A).
Meanwhile, DSS administration for 7 days led to severe diarrhea, blood in stool and body weight loss integrated as remarkably high disease activity index compared with normal group $(p<0.01)$, while this elevation was relieved by GQD and SASP treatment $(p<0.01$, Figure 8B). In addition, after the establishment of UC model, DSS resulted in a significant shortening of the colon length, while GQD and SASP treatment markedly reduced DSS-induced colon shortening compared with model group $(p<0.01$, Figure $8 \mathrm{CC}$ ). Collectively, we assessed preliminary effect of GQD on UC, and the results suggested that GQD exerted protective effects in UC treatment.

\section{Effects of Gegen Qinlian Decoction on Histopathological Changes in Colon Tissues}

The diarrhea and stool blood caused by daily DSS administration were accompanied by inflammation in colonic tissues and damage to the intestinal wall. HE staining was applied to observe the pathological changes of intestinal tissue in each group. As shown in Figure 9, the colonic mucosa was intact, and the intestinal epithelial cells and glands were arranged neatly in control group. And severe epithelial cell necrosis, crypt 
TABLE 2 | Potential targets of GQD on UC.

\begin{tabular}{|c|c|c|c|}
\hline Gene name & Target & Uniprot ID & Degree \\
\hline ESR1 & Estrogen receptor alpha & P03372 & 47 \\
\hline EGFR & Epidermal growth factor receptor erbB1 & P00533 & 38 \\
\hline LCK & Tyrosine-protein kinase LCK & P06239 & 29 \\
\hline SRC & Tyrosine-protein kinase SRC & P12931 & 27 \\
\hline KDR & Vascular endothelial growth factor receptor 2 & P35968 & 27 \\
\hline MMP2 & Matrix metalloproteinase 2 & P08253 & 26 \\
\hline GSK3 $\beta$ & Glycogen synthase kinase- 3 beta & P49841 & 26 \\
\hline PTGS2 & Cyclooxygenase-2 & P35354 & 25 \\
\hline MMP9 & Matrix metalloproteinase 9 & P14780 & 24 \\
\hline HSP90AA1 & Heat shock protein HSP 90-alpha & P07900 & 23 \\
\hline $\mathrm{AR}$ & Androgen receptor & P10275 & 23 \\
\hline ABL1 & Tyrosine-protein kinase ABL & P00519 & 23 \\
\hline PARP1 & Poly [ADP-ribose] polymerase-1 & P09874 & 22 \\
\hline MTOR & Serine/threonine-protein kinase mTOR & P42345 & 17 \\
\hline MCL1 & Induced myeloid leukemia cell differentiation protein Mcl-1 & Q07820 & 17 \\
\hline RPSKB1 & Ribosomal protein S6 kinase 1 & P23443 & 17 \\
\hline PIK3CA & Pl-kinase p110-alpha subunit & P42336 & 16 \\
\hline CDK4 & Cyclin-dependent kinase 4 & P11802 & 16 \\
\hline PPARG & Peroxisome proliferator-activated receptor gamma & P37231 & 15 \\
\hline IL2 & Interleukin-2 & P60568 & 14 \\
\hline MAPK14 & MAP kinase p38 alpha & Q16539 & 13 \\
\hline MAPK8 & c-Jun $\mathrm{N}$-terminal kinase 1 & P45983 & 12 \\
\hline PIK3R1 & PI3-kinase p85-alpha subunit & P27986 & 12 \\
\hline JAK2 & Tyrosine-protein kinase IAK2 & 060674 & 12 \\
\hline MAPK2K1 & Dual specificity mitogen-activated protein kinase 1 & Q02750 & 11 \\
\hline IGF1R & Insulin-like growth factor 1 receptor & P08069 & 11 \\
\hline MPO & Myeloperoxidase & P05164 & 10 \\
\hline RAC1 & Ras-related C3 botulinum toxin substrate 1 & P63000 & 9 \\
\hline MAPK1 & MAP kinase ERK2 & P28482 & 8 \\
\hline EP300 & Histone acetyltransferase p300 & Q09472 & 8 \\
\hline CDC42 & Cell division control protein 42 homolog & P60953 & 8 \\
\hline $\mathrm{F} 2$ & Thrombin & P00734 & 8 \\
\hline SIRT1 & NAD-dependent control protein 42 homolog & Q96EB6 & 6 \\
\hline RELA & Nuclear factor NF-kappa-B p65 subunit & Q04206 & 6 \\
\hline AKT1 & Serine/threonine-protein kinase AKT & P31749 & 5 \\
\hline TNF & Tumor necrosis factor & P01375 & 5 \\
\hline CASP3 & Caspase-3 & P42574 & 5 \\
\hline MDM2 & P53-binding protein Mdm-2 & Q00987 & 5 \\
\hline FYN & Tyrosine-protein kinase fyn & P06241 & 5 \\
\hline $\mathrm{NR} 3 \mathrm{C} 1$ & Glucocorticoid receptor & P04150 & 5 \\
\hline PTK2 & Focal adhesion kinase 1 & Q05397 & 5 \\
\hline ERBB2 & Receptor protein-tyrosine kinase erbB-2 & P04626 & 4 \\
\hline BCL2L1 & Apoptosis regulator $\mathrm{Bcl}-\mathrm{X}$ & Q07817 & 4 \\
\hline STAT3 & Signal transducer and activator pf transcription 3 & P40763 & 3 \\
\hline ICAM1 & Intercellular adhesion molecule-1 & P05362 & 3 \\
\hline$N F_{\kappa} B$ & Nuclear factor NF-kappa-B p105 subunit & P19838 & 3 \\
\hline PTPN11 & Protein-tyrosine phosphatase 2C & Q06124 & 3 \\
\hline SERPPINE1 & Plasminogen activator inhibitor-1 & P05121 & 3 \\
\hline STAT6 & Signal transducer and activator of transcription 6 & P42226 & 3 \\
\hline VEGFA & Vascular endothelial growth factor A & P15692 & 2 \\
\hline MAPK3 & MAP kinase ERK1 & P27361 & 2 \\
\hline CXCR4 & C-X-C chemokine receptor type 4 & P61073 & 2 \\
\hline STAT1 & Signal transducer and activator of transcription 1 alpha/beta & P42224 & 2 \\
\hline HIF1A & Hypoxia-inducible factor 1 alpha & Q16665 & 2 \\
\hline $\mathrm{XIAP}$ & Inhibitor of apoptosis protein 3 & P98170 & 2 \\
\hline FGF2 & Basic fibroblast growth factor & P09038 & 1 \\
\hline ATM & Serine-protein kinase ATM & Q13315 & 1 \\
\hline TGFB1 & Transforming growth factor & P01137 & 1 \\
\hline PRKCD & Protein kinase $\mathrm{C}$ delta & Q05655 & 1 \\
\hline
\end{tabular}

destruction and inflammatory cell infiltration were observed in DSS-treated mice. However, both GQD and SASP treatment could attenuate the histopathological manifestation of colitis.
Mild epithelial cells degeneration or necrosis, and a small number of inflammatory cells could be observed in GQD and SASP group. 


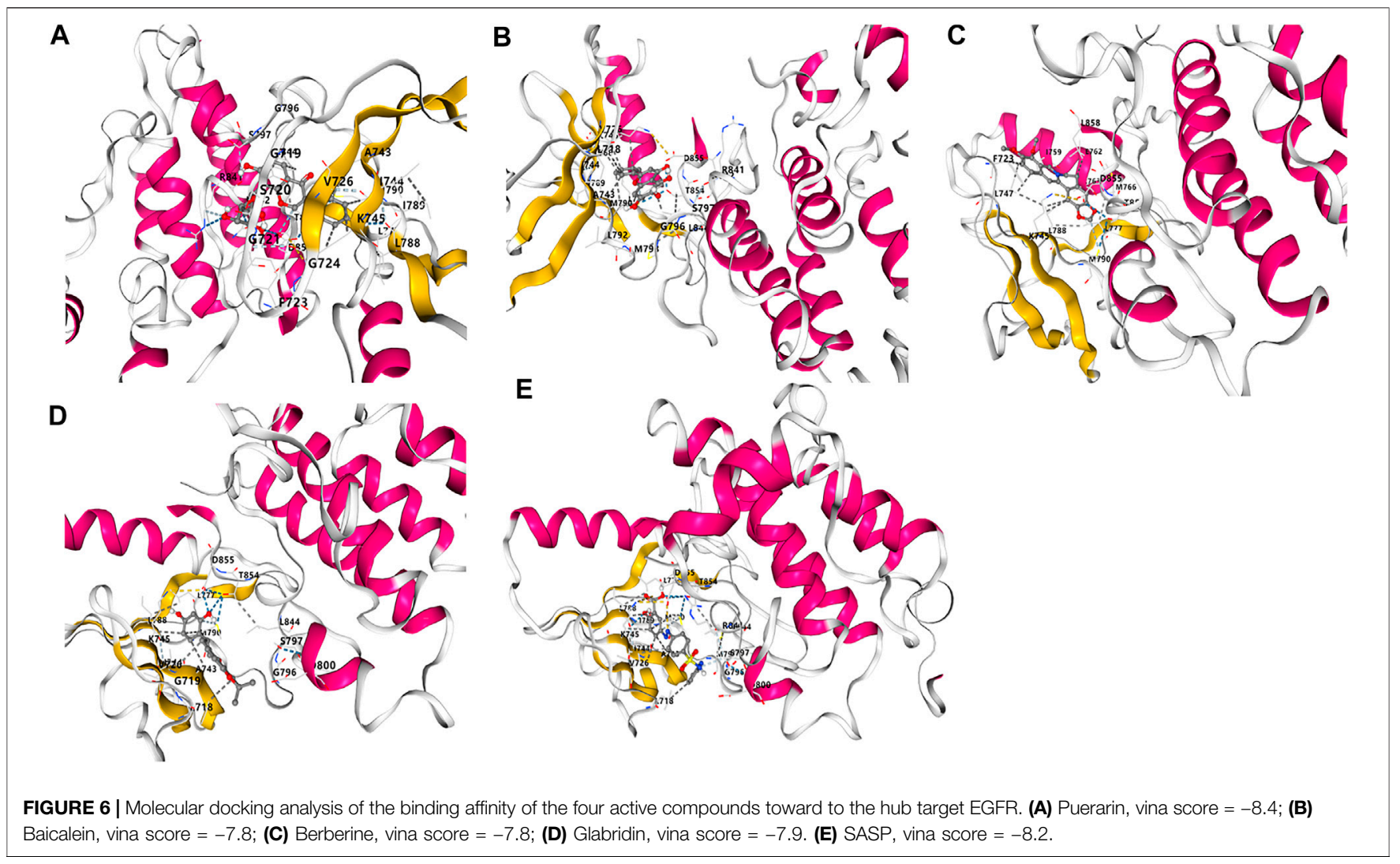

\section{Gegen Qinlian Decoction-Treatment Inhibited the Pro-Inflammatory Cytokines in Colon Tissues}

As pro-inflammatory factors play important roles in the pathogenesis and progress of UC, we investigated whether GQD possess the anti-inflammatory effect in UC. ELISA essay was performed in colonic samples of all the experimental groups. As shown in Figure 10, DSS significantly evoked the expression of pro-inflammatory cytokines including TNF- $\alpha$, IL- $1 \beta$, and IL- 6 compared with those in sham group $(p<0.01)$. However, the administration of GQD and SASP inhibited the content of TNF$\alpha$, IL- $1 \beta$, and IL- 6 significantly than that of the model group $(p<$ $0.01)$. These data demonstrated that GQD and SASP could inhibit the inflammatory reaction induced by DSS in UC.

\section{Gegen Qinlian Decoction Ameliorated Dextran Sulfate Sodium-Induced Inflammation Through EGFR/PI3K/AKT Signaling Pathway}

Based on numerous studies and the network pharmacology analysis, inflammation plays an essential role in the pathogenesis and process of $\mathrm{UC}$, and the inflammatory response may partially mediate by EGFR/PI3K/AKT signaling pathway (Neurath, 2014). Thereby, we investigated whether GQD could exert therapeutic effects on UC by inhibiting inflammation via regulating the expression of EGFR, PI3K, and $p$-AKT. As shown in Figure 11, western blotting results showed that DSS induced a remarkably increase in the protein expression of EGFR, PI3K, and $p$-AKT in colon tissue, which indicates the activation of EGFR/PI3K/AKT signaling pathway in UC. Notably, the elevation of these protein targets was blocked by GQD treatment. Similarly, administration of SASP downregulated the protein expression of EGFR, PI3K, and p-AKT as well. These results demonstrated that GQD protects against UC partially through inhibiting the activation of EGFR/ PI3K/AKT signaling pathway.

\section{DISCUSSION}

UC is a common intestinal inflammatory disease. Presently, numerous studies have demonstrated that GQD, as a classical complementary and alternative TCM prescription, exerted acclaimed therapeutic effects against UC (Xu et al., 2015; Li et al., 2016). Whereas, these clinical or experimental trials were designed on the basis of traditional research model of "one drug, one target", ignoring the complexity of biological systems. It is well known that botanical medicines usually contain a large group of chemical components, which may act synergistically to achieve the therapeutic effects (Qiao et al., 2016). Thus, to uncover the underlying mechanisms of the therapeutic effects of GQD on UC in a systemic perspective, the present study employed network pharmacological strategy. By means of active compounds screening, drug targeting, and pathway enrichment analysis, 


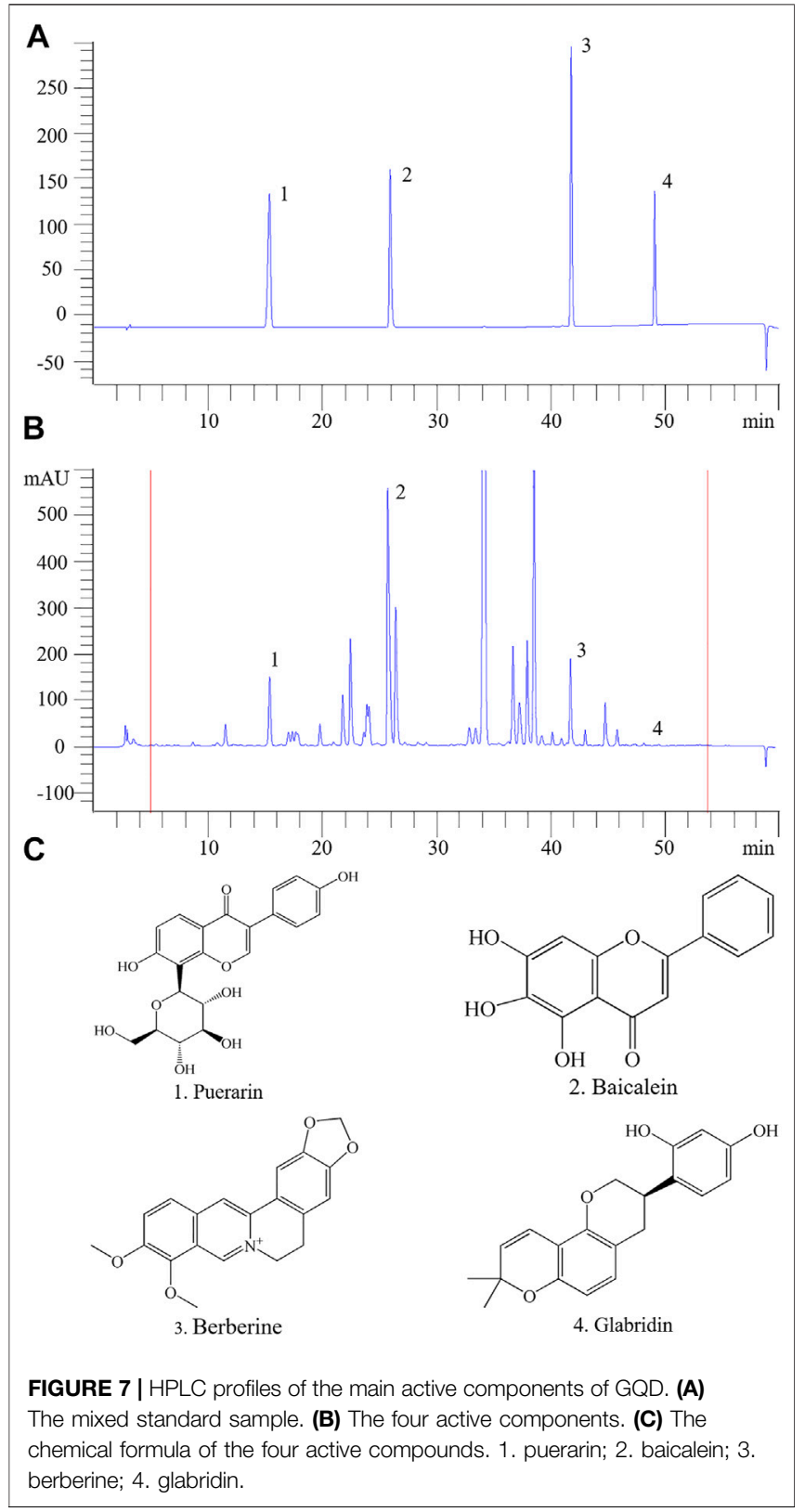

network pharmacological analysis of GQD identified four botanical drugs, 77 compounds, and 59 hub targets, which were significantly enriched in several pathways related to UC such as HIF-1 signaling pathway, PI3K-AKT signaling pathway and TNF signaling pathway. In addition, molecular docking results demonstrated that the crucial active compounds in GQD exhibited good binding affinity to the hub target. The putative active components and multi-targets mechanisms of GQD in the treatment of UC were comprehensively elucidated in the present study, which provided theoretic evidence for the clinical application of GQD on UC treatment.

Among the identified compounds linked to the network, most chemical ingredients in GQD related to UC could be classified into flavonoids, alkaloids, and triterpenoid saponins. Baicalein and wogonin (a flavonoid), the major bioactive compounds isolated from HQ, had a better absorption effect. It has been reported that baicalein possesses potent anti-inflammatory and anti-colitis effect via suppressing the activity of TNF- $\alpha$ and interleukin IL-1 $\beta$ (He et al., 2015; Luo et al., 2017), as well as inhibiting the expression of NF- $\mathrm{BB}$ and STAT3 signaling pathways (Dong et al., 2015), while the wogonin exerts a dramatically preventive effect on colitis-associated cancer with its anti-angiogenesis activity through inhibiting secretion of VEGF and accelerated the degradation of HIF-1a (Song et al., 2013; Sun et al., 2015). Additionally, formononetin (an isoflavone) was also recognized as one of potential ingredients in the treatment of $\mathrm{UC}$, which might possess the prominent antiinflammation and antiproliferative effect on human colorectal cancer by downregulating the expression of HIF-1a and inflammatory cytokines (Huang et al., 2015; Ong et al., 2019), such as TNF- $\alpha$, NF- $\kappa$ B (Wang et al., 2012). Glabridin, another isoflavonoid retrieved from GC, associated with a wide range of biological properties such as antioxidant, anti-inflammatory and antibacterial activities (Simmler et al., 2013). In addition, berberine possessed relatively high bioactivity and showed the high degree number of interactions linked to hub targets. It was a quaternary ammonium alkaloid from HL that exerted beneficial effects against UC via suppressing the IL-6/STAT3/NF- $\mathrm{B}$ signaling pathway in vivo (Zhang et al., 2017; Zhu et al., 2019). According to previous research, the anti-inflammatory effect of berberine was mediated through COX-2 regulation (Kuo et al., 2004). Epiberberine is also a protoberberine alkaloid with antibacterial activities and synergistic effects for berberine (Luo et al., 2013; Tan et al., 2017). Moreover, puerarin, one of the main isoflavonoid components in Pueraria lobata (Willd.) Ohwi, has been known to possess anti-inflammatory and antioxidant effects in UC via down-regulating the expression of nuclear factor- $\mathrm{kB}$ and the secretion of pro-inflammatory mediators (Jeon et al., 2020). Overall, the above findings indicate that multi-compounds of GQD may conjointly execute the beneficial effects for UC. And the HPLC results in the present study also showed the existence and contents of puerarin, baicalein, berberine, and glabridin in GQD, which provided the pharmacological evidence for GQD to be used as an agent in the UC treatment. Besides the therapeutic effects of important compounds mentioned above, multiple actions and pharmacological efficacy of comprehensive ingredients in GQD have not been well evaluated yet, which is a challenge in pharmacology study due to the complex composition of compounds, synergetic effects and multitargets of compounds in botanical drugs (Heinrich et al., 2020). And in the present study, numerous targets and pathways were identified to be relate to multiple compounds from different botanical drugs in GQD, which revealed the synergistic property of the compounds in GQD for the treatment of UC.

Among the main putative target mapped to compounds in the C-T network, many targets (ESR1, EGFR, SRC, MMP2, GSK3B, and PTGS2) with higher degree to compound might play a crucial role in the process of UC treatment. ESR1, an estrogen receptor, has been identified as a potential marker for identifying individuals at increased risk of neoplasia among those with 


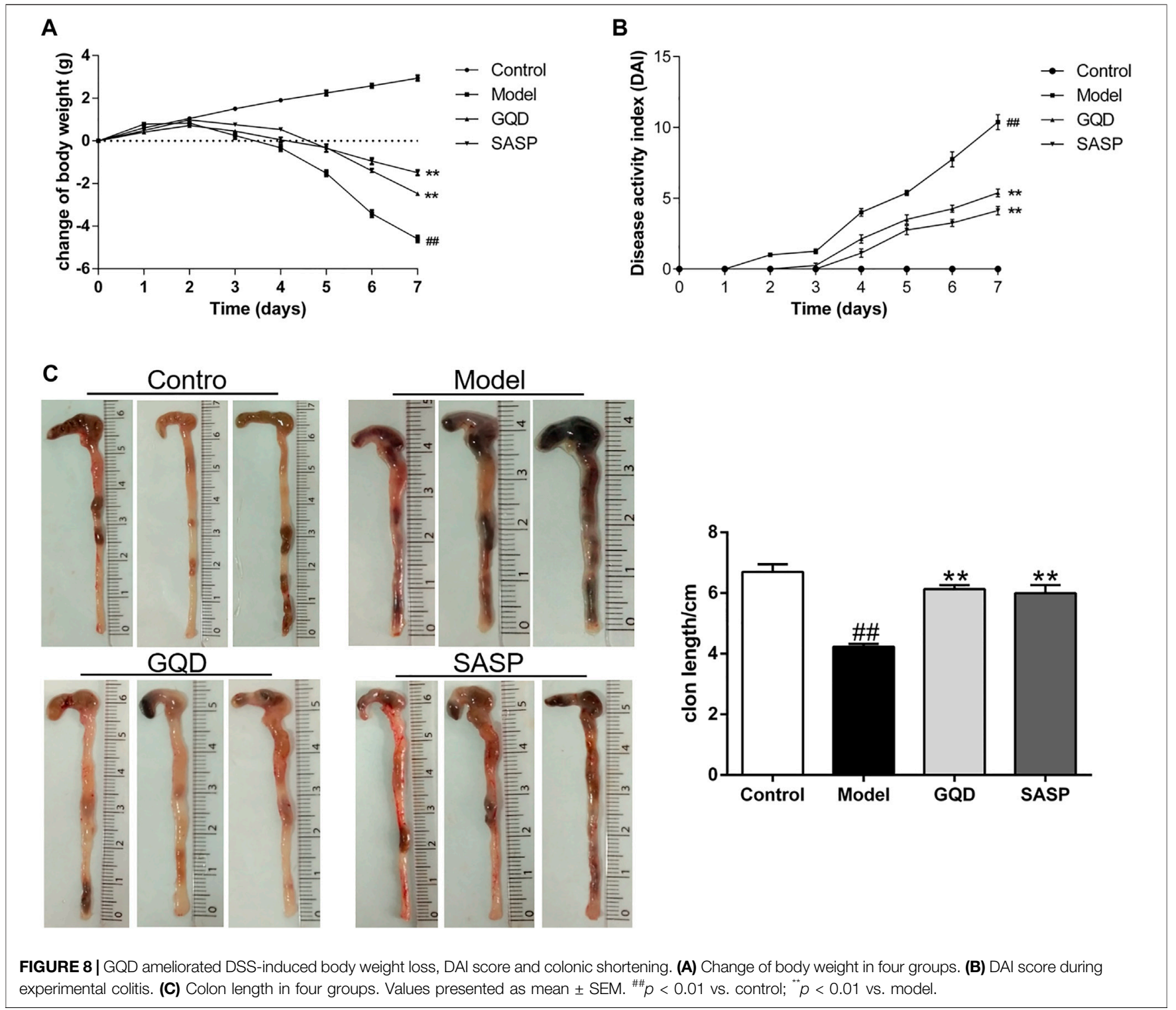

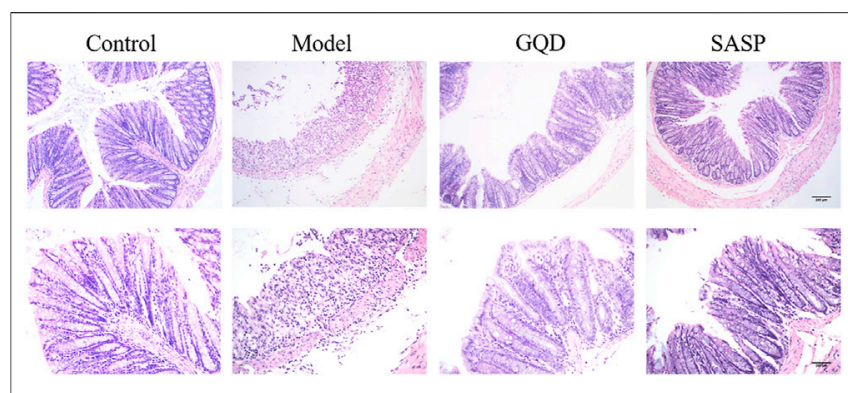

FIGURE 9 | Representative images of colonic tissues with HE staining ( $\times 100$ and $\times 200$ magnification).

long-standing and extensive UC (Principi et al., 2014). EGFR, the epidermal growth factor receptor, is one of the major proteins regulating cell proliferation and survival on gut mucosal (Yarden and Pines, 2012). The binding of EGF at the enterocyte surface induces the dimerization of EGFR and tyrosine autophosphorylation, of which the formation initiates several intracellular signaling pathways including PI3K/AKT signaling pathway, and Ras/ERK signaling pathway (Wang, 2017). The GSK3B, as the components of the PI3K pathway, also can be initiated by chronic inflammation and the increased turnover of epithelial cells in the development of UC (Rogler, 2014; Setia et al., 2014). And the expression level of main target MMP2 also has been found to be closely related with TNF- $\alpha$ in colonic mucosa subjected to UC inflammation (Mao et al., 2012). In this study, we speculated that above targets closely related to GQD may be important functional targets for the treatment of UC. Besides, according to GO enrichment and KEGG pathway enrichment, the network analysis revealed that the GQD may exert a therapeutic effect to UC via many cellular processes, such as the regulation of inflammatory response, cell proliferation, 

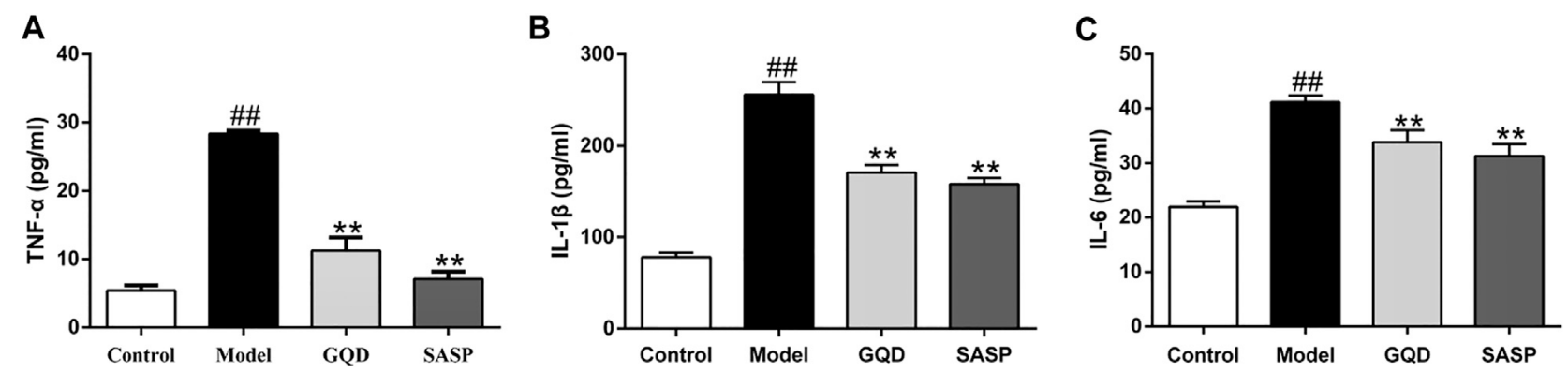

FIGURE 10 | The influence of GQD and SASP on proinflammatory cytokines TNF $\alpha$ (A), IL-1 $\beta$ (B), and IL-6 (C) in the colonic tissues. Values presented as mean \pm SEM. ${ }^{\# \#} p<0.01$ vs. control; ${ }^{* *} p<0.01$ vs. model.

angiogenesis and apoptosis. Moreover, among the enriched pathways linked to the hub targets, multiple pathways, such as PI3K-AKT signaling pathway, HIF-1 signaling pathway, VEGF signaling pathway, TNF signaling pathway, and Ras signaling pathway may be closely related to the pathogenesis of UC.

Dysregulated inflammatory response has been demonstrated to play a crucial role in the initiation, development, and progression of UC (Neurath, 2014). Inflammation and inflammatory cytokine in intestinal epithelial cells, and inflammatory cells were recognized as a risk factor for chronic inflammation to tumorigenesis (Yao et al., 2019). Moreover, colorectal cancer is a well-recognized complication of UC. And patients with long-standing chronic inflammation have an increased risk of potential carcinogenesis (Rubin et al., 2012). In UC, the disturbed intestinal epithelial cell generated the mucosal barrier dysfunction and the development of ulcers, leading to cancer occurrence (Yang et al., 2009). In the network pharmacology study, we found that many active components, prediction targets and pathways related to GQD were involved in the inflammation, angiogenesis, cell proliferation and apoptosis, which are closely related to cell carcinogenesis in UC. Specifically, the role of EGFR in inflammatory pathologies of colon is well known, and the expression of EGFR has been found upregulate in experimental colitis and patients with UC, then regulates cytokines production and intestinal inflammation ( $\mathrm{Lu}$ et al., 2014). Moreover, PI3K is one of the EGFR targets, and the abnormality of PI3K/AKT signaling pathway is considered to be related to the onset of UC, as well as UC-induced colon cancer (Jiang et al., 2019). PI3K-AKT pathway is tightly related to the regulation of inflammatory response in the progression of UC. The abnormal activation of PI3K/AKT signaling pathway in UC has been demonstrated to enhance the expression and secretion of proinflammatory cytokines, such as TNF- $\alpha$, IL- $1 \beta$, and IL-6 (Wu et al., 2013; Jiang et al., 2019). However, TNF-a is a proinflammatory cytokine central to the pathogenesis of UC, which has a direct effect on the expression and organization of tight junction proteins, resulting in loss of tight junction functions, increased epithelial permeability and the induction of an inflammatory response (Leppkes et al., 2014). Hence, anti-TNF therapies is widely used in clinic as an appropriate way in the management of UC (Lawrance, 2015). Especially, TNF signaling pathway exerts a broad spectrum of activities, including regulation of inflammation and the migration of intestinal epithelial cells (Armaka et al., 2008; Schliemann et al., 2011), and it has been confirmed that ErbB signaling can be activated by the phosphorylation of TNF- $\alpha$-induced receptor, which modulates the intestinal wound healing of inflammatory bowel disease (Frey et al., 2009). And PI3K/AKT signaling pathway is active in cells infiltrating inflamed human colon tissue via the participation of PTEN, GSK3B and mTOR, which may lead to over-transcription of downstream target genes, proliferation of intestinal epithelial cells, and even dysplasia and cancerization (Castellano and Downward, 2011; Matsuda et al., 2013; Chen et al., 2015). In addition, HIF-1, a transcription factor that regarded as the master regulator of oxygen homeostasis, is involved in the expression of a broad genetic program, including toll-like receptors (TLR)-mediated inflammatory pathway, which can trigger the activation of transcription factors (such as NF- $\mathrm{B}$ ) and induce TNF-a secretion (Jung et al., 2003; Kim et al., 2012). It has been reported that HIF-1 play an important role in vascular remodeling at sites of intestinal injury and inflammation of IBD, by regulating angiogenic genes such as VEGF (Feinman et al., 2010; Bakirtzi et al., 2014), a major paracrine growth factor involved in blood vessel development, which has been unregulated in active UC and UC-associated cancer (Tolstanova et al., 2009). Meanwhile, the fundamental regulator of angiogenesis and vascular permeability of VEGF has been found, which indicates that VEGF may also has proinflammatory properties (Chidlow et al., 2006). VEGF signaling pathway can be promoted by increased many inflammatory cells infiltrating ulcerative mucosa. And the increased leukocyte infiltration would contribute to inflammation initiation and subsequent tissue damage which is a characteristic feature of UC (Goebel et al., 2006). Therefore, we hypothesize that GQD may exert its therapeutic effects on UC by decreasing the inflammation response via regulating EGFR/PI3K/ AKT signaling pathway.

To examine the effect of GQD on the inflammatory response in UC, as well as the role of EGFR-regulated PI3K/AKT signaling pathway in DSS-induced UC, we evaluated colon inflammation and injury in mice administrated with DSS. DSS-treated mice in model group exhibited more severe injury and acute colitis, while these abnormalities were ameliorated by GQD or SASP administration. We found that 

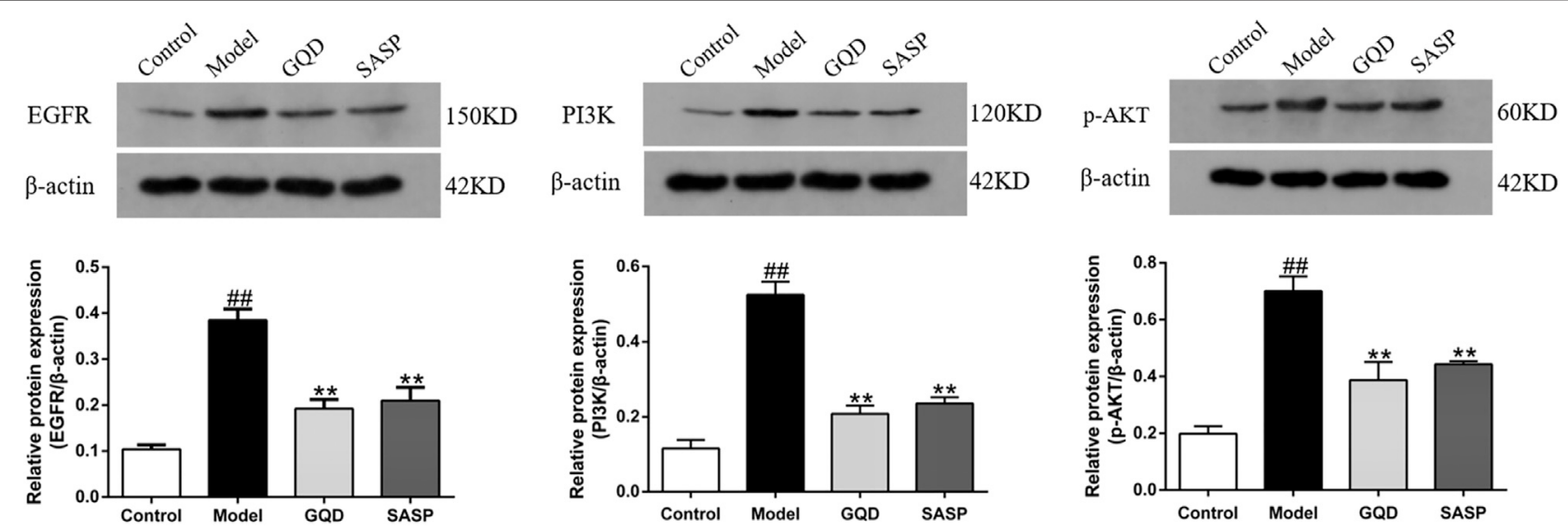

FIGURE 11 | Western blot and quantitative analysis of EGFR, PI3K and $p$-AKT in the colon tissues. Values presented as mean \pm SEM. ${ }^{\# \#} p<0.01$ vs. control; ${ }^{* *} p<$ 0.01 vs. model.

GQD and SASP could alleviate the shortening of the colon length, the DAI parameters, the pathological changes and the secretion of inflammatory cytokines. Moreover, the results indicated that EGFR/PI3K/AKT signaling pathway was activated abnormally in model group and played an important role in inflammation, consequently, a series of inflammatory responses and mucosal damage occurs, resulting in the development of UC. However, the expression of EGFR, PI3K and $p$-AKT in the colon tissues was significantly decreased in the GQD and SASP groups compared to model group, suggesting the protective effects of GQD and SASP in the treatment of UC. From the integrated drug target prediction, pathway enrichment analysis and in vivo experiment, the results demonstrated that the effective downregulation of the expression of EGFR, PI3K, and $p$-AKT by the administration of GQD and SASP may contribute to ameliorating UC.

The limitation of this study is lacking standardization of active compounds in GQD. However, it has been demonstrated that the governing authorities' rules on market entry of TCM products is primarily focused on the quality, safety and efficacy of TCM (Li et al., 2018). Compared with chemical drugs and biological products, the quality control of TCM is much more complicated and difficult because of the extremely large amounts of bioactive components and diverse synergistic effects. Standardization of TCM is an essential part of the modernization of TCM, which has been a topic of discussion over the past few decades in China with the goal to promote advances in TCM (Song et al., 2011). With regards to quality, international consensus is that all the TCM products must meet certain qualitative and quantitative quality standards that demonstrated authentication, identification and chemical composition (Qu et al., 2019), and several national standards of TCM has been proposed in China over the past few decades, Chinese Pharmacopoeia is the cornerstone of national standards for TCM (Song et al., 2011). The considerations for quality control should mainly include the following aspects: the source control of natural raw material, standardization of decoction pieces processing, inprocess control of manufacturing, and specification that fit the characteristics of TCM. In addition, for the selection of quantitative control indicators, the active ingredients that relate to clinical function indications is considered, and when the active ingredients are unclear, representative components should be chosen ( $\mathrm{Qu}$ et al., 2019). Development of the qualitative and quantitative analytic method to capture the fingerprint and content information of multiple chemical markers is the basis for standardization of TCM. Recently, a concept of "quality marker" (Q-marker) was proposed. Q-marker-base fingerprint and multicomponent determination could contribute to the construction of more scientific quality control system of TCM (Yang et al., 2017). TCM standardization is an inevitable trend for the purpose of comprehensive development of TCM, and many measures should be taken to further promote the standardization of TCM.

Most recently, the draft of Network Pharmacology Evaluation Method Guidance was proposed, which addressed the data collection, network analysis, experiment verification principles, procedures, and evaluation indicators in network pharmacology research (Li, 2021). According to the guidance, there are multiple problems in the current network pharmacology studies, such as uneven research quality, lack of data standardization, and insufficient scientific verification. Here, we use the evaluation methods from three aspects-reliability, standardization, and rationality to assess the quality of our present study and find that it basically meets the evaluation requirement. Biological functional prediction is an important part in network pharmacology. The inflammatory response, regulation of apoptotic process, protein phosphorylation, and cell proliferation have been identified as important biological functions according to our prediction, while we mainly focused on inflammatory response in this paper, and in the future we will carry out further experiments to probe other possible predicted functions and mechanisms.

\section{CONCLUSION}

In summary, the combination of network pharmacological analysis in silico and in vivo experiment indicated that 
GQD could effectively ameliorate DSS-induced colitis, and the therapeutic effect of GQD was associated with its synergic modulation on inflammation suppression via EGFR/PI3K/ AKT signaling pathway. Therefore, we suggest that GQD could be developed as a promising therapy for UC, and it provided novel indications for further mechanistic studies of GQD on UC treatment. Our study also suggested that the network pharmacology prediction may exert as a useful tool to characterize the pharmacological mechanisms of TCM in detail.

\section{DATA AVAILABILITY STATEMENT}

The raw data supporting the conclusions of this article will be made available by the authors, without undue reservation, to any qualified researcher.

\section{ETHICS STATEMENT}

The animal study was reviewed and approved by Laboratory Animal Ethical Committee of China Three Gorges University.

\section{REFERENCES}

Armaka, M., Apostolaki, M., Jacques, P., Kontoyiannis, D. L., Elewaut, D., and Kollias, G. (2008). Mesenchymal Cell Targeting by TNF as a Common Pathogenic Principle in Chronic Inflammatory Joint and Intestinal Diseases. J. Exp. Med. 205, 331-337. doi:10.1084/jem.20070906

Artursson, P., and Karlsson, J. (1991). Correlation between Oral Drug Absorption in Humans and Apparent Drug Permeability Coefficients in Human Intestinal Epithelial (Caco-2) Cells. Biochem. Biophysical Res. Commun. 175, 880-885. doi:10.1016/0006-291x(91)91647-u

Awortwe, C., Fasinu, P. S., and Rosenkranz, B. (2014). Application of Caco-2 Cell Line in Herb-Drug Interaction Studies: Current Approaches and Challenges. J. Pharm. Pharm. Sci. 17, 1-19. doi:10.18433/j30k63

Bakirtzi, K., West, G., Fiocchi, C., Law, I. K. M., Iliopoulos, D., and Pothoulakis, C. (2014). The Neurotensin-HIF-1 $\alpha$-Vegfa Axis Orchestrates Hypoxia, Colonic Inflammation, and Intestinal Angiogenesis. Am. J. Pathol. 184, 3405-3414. doi:10.1016/j.ajpath.2014.08.015

Bressler, B., Marshall, J. K., Bernstein, C. N., Bitton, A., Jones, J., Leontiadis, G. I., et al. (2015). Clinical Practice Guidelines for the Medical Management of Nonhospitalized Ulcerative Colitis: The Toronto Consensus. Gastroenterology 148, 1035-1058. doi:10.1053/j.gastro.2015.03.001

Castellano, E., and Downward, J. (2011). RAS Interaction with PI3K: More Than Just Another Effector Pathway. Genes. Cancer 2, 261-274. doi:10.1177/ 1947601911408079

Chen, J., Shao, R., Li, F., Monteiro, M., Liu, J.-P., Xu, Z. P., et al. (2015). PI3K/Akt/ mTOR Pathway Dual Inhibitor BEZ235 Suppresses the Stemness of colon Cancer Stem Cells. Clin. Exp. Pharmacol. Physiol. 42, 1317-1326. doi:10.1111/ 1440-1681.12493

Chidlow, J. H., Langston, W., Greer, J. J. M., Ostanin, D., Abdelbaqi, M., Houghton, J., et al. (2006). Differential Angiogenic Regulation of Experimental Colitis. Am. J. Pathol. 169, 2014-2030. doi:10.2353/ajpath.2006.051021

Chow, S.-C. (2014). Bioavailability and Bioequivalence in Drug Development. Wiley Interdiscip. Rev. Comput. Stat. 6, 304-31. doi:10.1002/wics.1310

Dong, S.-j., Zhong, Y.-q., Lu, W.-t., Li, G.-h., Jiang, H.-l., and Mao, B. (2015). Baicalin Inhibits Lipopolysaccharide-Induced Inflammation through Signaling NF-Kb Pathway in HBE16 Airway Epithelial Cells. Inflammation 38, 1493-1501. doi:10.1007/s10753-015-0124-2

\section{AUTHOR CONTRIBUTIONS}

$\mathrm{ZM}$ and YF conceived and designed the study; XL and YF performed the study, analyzed the data; XL and ZM drafted the manuscript; LD provided some positive suggestions and amended the final manuscript; $\mathrm{XL}$ and $\mathrm{LD}$ contributed to molecular docking analysis; $\mathrm{ZM}$ and YF contributed to revising the manuscript and improving the language in the final version of manuscript.

\section{FUNDING}

This work was supported by the fund for Youth Top Talent Project of Hubei Provincial Health and Family Planning Commission (EWT-2019-48), and Key Projects of Hunan Province Education Department (20A366).

\section{ACKNOWLEDGMENTS}

Special thanks to Xiang Guo and Tong Yang for the assistance in the network pharmacology approach and molecular docking analysis.

Fan, Y., Yi, W., Huang, H., Mei, Z., and Feng, Z. (2019). Efficacy of Herbal Medicine (Gegen Qinlian Decoction) on Ulcerative Colitis. Medicine (Baltimore) 98 (52), e18512. doi:10.1097/MD.0000000000018512

Feinman, R., Deitch, E. A., Watkins, A. C., Abungu, B., Colorado, I., Kannan, K. B., et al. (2010). HIF-1 Mediates Pathogenic Inflammatory Responses to Intestinal Ischemia-Reperfusion Injury. Am. J. Physiology-Gastrointestinal Liver Physiol. 299, G833-G843. doi:10.1152/ajpgi.00065.2010

Frey, M. R., Edelblum, K. L., Mullane, M. T., Liang, D., and Polk, D. B. (2009). The ErbB4 Growth Factor Receptor Is Required for colon Epithelial Cell Survival in the Presence of TNF. Gastroenterology 136, 217-226. doi:10.1053/j.gastro.2008.09.023

Gallo, G., Kotze, P. G., and Spinelli, A. (2018). Surgery in Ulcerative Colitis: when? How? Best Pract. Res. Clin. Gastroenterol. 32-33, 71-78. doi:10.1016/j.bpg.2018.05.017

Gfeller, D., Grosdidier, A., Wirth, M., Daina, A., Michielin, O., and Zoete, V. (2014). SwissTargetPrediction: a Web Server for Target Prediction of Bioactive Small Molecules. Nucleic Acids Res. 42, W32-W38. doi:10.1093/nar/gku293

Goebel, S., Huang, M., Davis, W. C., Jennings, M., Siahaan, T. J., Alexander, J. S. et al. (2006). VEGF-A Stimulation of Leukocyte Adhesion to Colonic Microvascular Endothelium: Implications for Inflammatory Bowel Disease. Am. J. Physiology-Gastrointestinal Liver Physiol. 290, G648-G654. doi:10.1152/ ajpgi.00466.2005

Hassan, S. M., and Hassan, A. H. (2018). The Possibility of Using Shogaol for Treatment of Ulcerative Colitis. Iran J. Basic Med. Sci. 21, 943-949. doi:10. 22038/IJBMS.2018.28616.6932

He, X., Wei, Z., Zhou, E., Chen, L., Kou, J., Wang, J., et al. (2015). Baicalein Attenuates Inflammatory Responses by Suppressing TLR4 Mediated NF-Kb and MAPK Signaling Pathways in LPS-Induced Mastitis in Mice. Int. Immunopharmacology 28, 470-476. doi:10.1016/j.intimp.2015.07.012

Head, K. A., and Jurenka, J. S. (2003). Inflammatory Bowel Disease Part 1: Ulcerative Colitis-Ppathophysiology and Conventional and Alternative Treatment Options. Altern. Med. Rev. 8, 247-283. doi:10.1046/j.1360-0443. 2003.00426.x

Heinrich, M., Appendino, G., Efferth, T., Fürst, R., Izzo, A. A., Kayser, O., et al. (2020). Best Practice in Research - Overcoming Common Challenges in Phytopharmacological Research. J. Ethnopharmacology 246, 112230. doi:10. 1016/j.jep.2019.112230

Huang, C., Zheng, C., Li, Y., Wang, Y., Lu, A., and Yang, L. (2014). Systems Pharmacology in Drug Discovery and Therapeutic Insight for Herbal Medicines. Brief Bioinform 15, 710-733. doi:10.1093/bib/bbt035 
Huang, J., Xie, M., Gao, P., Ye, Y., Liu, Y., Zhao, Y., et al. (2015). Antiproliferative Effects of Formononetin on Human Colorectal Cancer via Suppressing Cell Growth In Vitro and In Vivo. Process Biochem. 50, 912-917. doi:10.1016/j. procbio.2015.03.001

Jeon, Y.-D., Lee, J.-H., Lee, Y.-M., and Kim, D.-K. (2020). Puerarin Inhibits Inflammation and Oxidative Stress in Dextran Sulfate Sodium-Induced Colitis Mice Model. Biomed. Pharmacother. 124, 109847. doi:10.1016/j. biopha.2020.109847

Jiang, W., Han, Y. P., Hu, M., Bao, X. Q., Yan, Y., and Chen, G. (2019). A Study on Regulatory Mechanism of miR-223 in Ulcerative Colitis through PI3K/AktmTOR Signaling Pathway. Eur. Rev. Med. Pharmacol. Sci. 23, 4865-4872. doi:10.26355/eurrev_201906_18074

Jung, Y.-J., Isaacs, J. S., Lee, S., Trepel, J., and Neckers, L. (2003). IL-1 $\beta$ Mediated Up-regulation of HIF-la via an NFkB/COX-2 Pathway Identifies HIF-1 as a Critical Link between Inflammation and Oncogenesis. FASEB. J. 17, 1-22. doi:10.1096/fj.03-0329fje

Kim, D.-S., Kim, S.-J., Kim, M.-C., Jeon, Y.-D., Um, J.-y., and Hong, S.-H. (2012). The Therapeutic Effect of Chelidonic Acid on Ulcerative Colitis. Biol. Pharm. Bull. 35, 666-671. doi:10.1248/bpb.35.666

Kim, J., Yoo, M., Shin, J., Kim, H., Kang, J., and Tan, A. C. (2018b). Systems Pharmacology-Based Approach of Connecting Disease Genes in Genome-wide Association Studies with Traditional Chinese Medicine. Int. J. Genomics 2018, 1-11. doi:10.1155/2018/7697356

Kim, S., Shoemaker, B. A., Bolton, E. E., and Bryant, S. H. (2018a). Finding Potential Multitarget Ligands Using PubChem. Methods Mol. Biol. 1825, 63-91. doi:10.1007/978-1-4939-8639-2_2

Kuo, C.-L., Chi, C.-W., and Liu, T.-Y. (2004). The Anti-inflammatory Potential of Berberine In Vitro and In Vivo. Cancer Lett. 203, 127-137. doi:10.1016/j.canlet. 2003.09.002

Lawrance, I. C. (2015). Early Investigational TNF Receptor Antagonists for the Treatment of Ulcerative Colitis. Expert Opin. Investig. Drugs 24, 761-768. doi:10.1517/13543784.2015.1020371

Leppkes, M., Roulis, M., Neurath, M. F., Kollias, G., and Becker, C. (2014). Pleiotropic Functions of TNF- in the Regulation of the Intestinal Epithelial Response to Inflammation. Int. Immunol. 26, 509-515. doi:10.1093/intimm/ dxu051

Li, B., Rui, J., Ding, X., and Yang, X. (2019). Exploring the Multicomponent Synergy Mechanism of Banxia Xiexin Decoction on Irritable Bowel Syndrome by a Systems Pharmacology Strategy. J. Ethnopharmacology 233, 158-168. doi:10.1016/j.jep.2018.12.033

Li, H., Zhao, L., Zhang, B., Jiang, Y., Wang, X., Guo, Y., et al. (2014). A Network Pharmacology Approach to Determine Active Compounds and Action Mechanisms of Ge-Gen-Qin-Lian Decoction for Treatment of Type 2 Diabetes. Evidence-Based Complement. Altern. Med. 2014, 1-12. doi:10.1155/ 2014/495840

Li, J., Zhu, J., Hu, H., Harnett, J. E., Lei, C. I., Chau, K. Y., et al. (2018). Internationalization of Traditional/Complementary Medicine Products: Market Entry as Medicine. Chin. Med. 13, 50. doi:10.1186/s13020-018-0209-6

Li, R., Chen, Y., Shi, M., Xu, X., Zhao, Y., Wu, X., et al. (2016). Gegen Qinlian Decoction Alleviates Experimental Colitis via Suppressing TLR4/NF-Kb Signaling and Enhancing Antioxidant Effect. Phytomedicine 23, 1012-1020. doi:10.1016/j.phymed.2016.06.010

Li, S. (2021). Network Pharmacology Evaluation Method Guidance - Draft. World J. Tradit Chi Med. 7, 146-154. doi:10.4103/wjtcm.wjtcm_11_21

Li, S., Zhang, Z. Q., Wu, L. J., Zhang, X. G., Wang, Y. Y., and Li, Y. D. (2007). Understanding ZHENG in Traditional Chinese Medicine in the Context of Neuro-Endocrine-Immune Network. IET Syst. Biol. 1, 51-60. doi:10.1049/ietsyb:20060032

Liu, Y., Grimm, M., Dai, W.-t., Hou, M.-c., Xiao, Z.-X., and Cao, Y. (2020). CBdock: a Web Server for Cavity Detection-Guided Protein-Ligand Blind Docking. Acta Pharmacol. Sin 41, 138-144. doi:10.1038/s41401-019-0228-6

Liu, Z., Guo, F., Wang, Y., Li, C., Zhang, X., Li, H., et al. (2016). BATMAN-TCM: a Bioinformatics Analysis Tool for Molecular mechANism of Traditional Chinese Medicine. Sci. Rep. 6, 21146. doi:10.1038/srep21146

Lu, N., Wang, L., Cao, H., Liu, L., Van Kaer, L., Washington, M. K., et al. (2014). Activation of the Epidermal Growth Factor Receptor in Macrophages Regulates Cytokine Production and Experimental Colitis. J. Immunol. 192, 1013-1023. doi:10.4049/jimmunol.1300133
Luo, J., Yan, D., Yang, M., Dong, X., and Xiao, X. (2013). Multicomponent Therapeutics of Berberine Alkaloids. Evidence-Based Complement. Altern. Med. 2013, 1-10. doi:10.1155/2013/545898

Luo, X., Yu, Z., Deng, C., Zhang, J., Ren, G., Sun, A., et al. (2017). Baicalein Ameliorates TNBS-Induced Colitis by Suppressing TLR4/MyD88 Signaling cascade and NLRP3 Inflammasome Activation in Mice. Sci. Rep. 7, 16374. doi:10.1038/s41598-017-12562-6

Lv, R., Qiao, W., Wu, Z., Wang, Y., Dai, S., Liu, Q., et al. (2014). Tumor Necrosis Factor Alpha Blocking Agents as Treatment for Ulcerative Colitis Intolerant or Refractory to Conventional Medical Therapy: a Meta-Analysis. PLoS One 9, e86692. doi:10.1371/journal.pone.0086692

Mao, J.-W., Tang, H.-Y., Tan, X.-Y., and Wang, Y.-D. (2012). Effect of Etiasa on the Expression of Matrix Metalloproteinase-2 and Tumor Necrosis Factor- $\alpha$ in a Rat Model of Ulcerative Colitis. Mol. Med. Rep. 6, 996-1000. doi:10.3892/mmr. 2012.1021

Matsuda, S., Nakanishi, A., Wada, Y., and Kitagishi, Y. (2013). Roles of PI3K/AKT/ PTEN Pathway as a Target for Pharmaceutical Therapy. Tomcj 7, 23-29. doi:10. 2174/1874104501307010023

Neurath, M. F. (2014). Cytokines in Inflammatory Bowel Disease. Nat. Rev. Immunol. 14, 329-342. doi:10.1038/nri3661

Ong, S., Shanmugam, M., Fan, L., Fraser, S., Arfuso, F., Ahn, K., et al. (2019). Focus on Formononetin: Anticancer Potential and Molecular Targets. Cancers 11, 611. doi:10.3390/cancers11050611

Ordás, I., Eckmann, L., Talamini, M., Baumgart, D. C., and Sandborn, W. J. (2012). Ulcerative Colitis. The Lancet 380, 1606-1619. doi:10.1016/S0140-6736(12) 60150-0

Principi, M., Barone, M., Pricci, M., De Tullio, N., Losurdo, G., Ierardi, E., et al. (2014). Ulcerative Colitis: from Inflammation to Cancer. Do Estrogen Receptors Have a Role?. World. J. Gastroentrol. 20, 11496-11504. doi:10. 3748/wjg.v20.i33.11496

Qi, L., Kai, D., An, M. A., and Jia, L. (2004). Analysis of Puerarin and Daidzein in Gegen Qinlian Preparations by High Performance Capillary Electrophoresis. Chin. J. Pharm. Anal. 24, 11-13. doi:10.1016/S0898-6568(03)00096-2

Qiao, X., Wang, Q., Wang, S., Kuang, Y., Li, K., Song, W., et al. (2018). A 42Markers Pharmacokinetic Study Reveals Interactions of Berberine and Glycyrrhizic Acid in the Anti-diabetic Chinese Medicine Formula GegenQinlian Decoction. Front. Pharmacol. 9, 622. doi:10.3389/fphar.2018.00622

Qiao, X., Wang, Q., Wang, S., Miao, W.-j., Li, Y.-j., Xiang, C., et al. (2016). Compound to Extract to Formulation: a Knowledge-Transmitting Approach for Metabolites Identification of Gegen-Qinlian Decoction, a Traditional Chinese Medicine Formula. Sci. Rep. 6, 39534. doi:10.1038/srep39534

Qu, J., Zhang, T., Liu, J., Su, Y., and Wang, H. (2019). Considerations for the Quality Control of Newly Registered Traditional Chinese Medicine in China: A Review. J. AOAC Int. 102, 689-694. doi:10.5740/jaoacint.18-0301

Rogler, G. (2014). Chronic Ulcerative Colitis and Colorectal Cancer. Cancer Lett. 345, 235-241. doi:10.1016/j.canlet.2013.07.032

Ru, J., Li, P., Wang, J., Zhou, W., Li, B., Huang, C., et al. (2014). TCMSP: a Database of Systems Pharmacology for Drug Discovery from Herbal Medicines. J. Cheminform 6, 13. doi:10.1186/1758-2946-6-13

Rubin, D. C., Shaker, A., and Levin, M. S. (2012). Chronic Intestinal Inflammation: Inflammatory Bowel Disease and Colitis-Associated colon Cancer. Front. Immun. 3, 107. doi:10.3389/fimmu.2012.00107

Sałaga, M., Zatorski, H., Sobczak, M., Chen, C., and Fichna, J. (2014). Chinese Herbal Medicines in the Treatment of IBD and Colorectal Cancer: a Review. Curr. Treat. Options. Oncol. 15, 405-420. doi:10.1007/s11864-014-0288-2

Schliemann, M., Bullinger, E., Borchers, S., Allgöwer, F., Findeisen, R., and Scheurich, P. (2011). Heterogeneity Reduces Sensitivity of Cell Death for TNF-Stimuli. BMC Syst. Biol. 5, 204. doi:10.1186/1752-0509-5-204

Setia, S., Nehru, B., and Sanyal, S. N. (2014). Upregulation of MAPK/Erk and PI3K/ Akt Pathways in Ulcerative Colitis-Associated colon Cancer. Biomed. Pharmacother. 68, 1023-1029. doi:10.1016/j.biopha.2014.09.006

Shannon, P., Markiel, A., Ozier, O., Baliga, N. S., Wang, J. T., Ramage, D., et al. (2003). Cytoscape: a Software Environment for Integrated Models of Biomolecular Interaction Networks. Genome Res. 13, 2498-2504. doi:10. 1101/gr.1239303

Shijun, Z. (2010). Clinical Research of Gegenqinlian Decoction in Treating Dampess and Heat Ulcerative Colitis. China J. Chin. Med. 25, 970-971. doi:10.16368/j.issn.1674-8999.2010.05.052 
Shivashankar, R., Tremaine, W. J., Harmsen, W. S., and Loftus, E. V. (2017). Incidence and Prevalence of Crohn's Disease and Ulcerative Colitis in Olmsted County, Minnesota from 1970 through 2010. Clin. Gastroenterol. Hepatol. 15, 857-863. doi:10.1016/j.cgh.2016.10.039

Shu, Z., He, W., Shahen, M., Guo, Z., Shu, J., Wu, T., et al. (2018). Clarifying of the Potential Mechanism of Sinisan Formula for Treatment of Chronic Hepatitis by Systems Pharmacology Method. Biomed. Pharmacother. 100, 532-550. doi:10. 1016/j.biopha.2018.02.047

Simmler, C., Pauli, G. F., and Chen, S.-N. (2013). Phytochemistry and Biological Properties of Glabridin. Fitoterapia 90, 160-184. doi:10.1016/j.fitote.2013. 07.003

Song, P., Gao, J., Kokudo, N., and Tang, W. (2011). Standardization of Traditional Chinese Medicine and Evaluation of Evidence from its Clinical Practice. Drug. Discov. Ther. 5 (6), 261-265. doi:10.5582/ddt.2011.v5.6.261

Song, X., Yao, J., Wang, F., Zhou, M., Zhou, Y., Wang, H., et al. (2013). Wogonin Inhibits Tumor Angiogenesis via Degradation of HIF-1 a Protein. Toxicol. Appl. Pharmacol. 271, 144-155. doi:10.1016/j.taap.2013.04.031

Sun, Y., Zhao, Y., Yao, J., Zhao, L., Wu, Z., Wang, Y., et al. (2015). Wogonoside Protects against Dextran Sulfate Sodium-Induced Experimental Colitis in Mice by Inhibiting NF-Kb and NLRP3 Inflammasome Activation. Biochem. Pharmacol. 94, 142-154. doi:10.1016/j.bcp.2015.02.002

Szklarczyk, D., Gable, A. L., Lyon, D., Junge, A., Wyder, S., Huerta-Cepas, J., et al. (2019). STRING V11: Protein-Protein Association Networks with Increased Coverage, Supporting Functional Discovery in Genome-wide Experimental Datasets. Nucleic Acids Res. 47 (D1), D607-D613. doi:10.1093/nar/gky1131

Tan, L., Li, C., Chen, H., Mo, Z., Zhou, J., Liu, Y., et al. (2017). Epiberberine, a Natural Protoberberine Alkaloid, Inhibits Urease of Helicobacter pylori and jack Bean: Susceptibility and Mechanism. Eur. J. Pharm. Sci. 110, 77-86. doi:10. 1016/j.ejps.2017.02.004

Tao, W., Xu, X., Wang, X., Li, B., Wang, Y., Li, Y., et al. (2013). Network Pharmacology-Based Prediction of the Active Ingredients and Potential Targets of Chinese Herbal Radix Curcumae Formula for Application to Cardiovascular Disease. J. Ethnopharmacology 145, 1-10. doi:10.1016/j.jep. 2012.09.051

Tian, S., Wang, J., Li, Y., Li, D., Xu, L., and Hou, T. (2015). The Application of In Silico Drug-Likeness Predictions in Pharmaceutical Research. Adv. Drug Deliv. Rev. 86, 2-10. doi:10.1016/j.addr.2015.01.009

Tolstanova, G., Khomenko, T., Deng, X., Chen, L., Tarnawski, A., Ahluwalia, A., et al. (2009). Neutralizing Anti-vascular Endothelial Growth Factor (VEGF) Antibody Reduces Severity of Experimental Ulcerative Colitis in Rats: Direct Evidence for the Pathogenic Role of VEGF. J. Pharmacol. Exp. Ther. 328, 749-757. doi:10.1124/jpet.108.145128

Torres, J., Billioud, V., Sachar, D. B., Peyrin-Biroulet, L., and Colombel, J.-F. (2012). Ulcerative Colitis as a Progressive Disease: the Forgotten Evidence. Inflamm. Bowel Dis. 18, 1356-1363. doi:10.1002/ibd.22839

Wang, C., Duan, X., Sun, X., Liu, Z., Sun, P., Yang, X., et al. (2016). Protective Effects of Glycyrrhizic Acid from Edible Botanical glycyrrhiza Glabra against Non-alcoholic Steatohepatitis in Mice. Food Funct. 7, 3716-3723. doi:10.1039/c6fo00773b

Wang, Y., Zhu, Y., Gao, L., Yin, H., Xie, Z., Wang, D., et al. (2012). Formononetin Attenuates IL-1 $\beta$-Induced Apoptosis and NF-Kb Activation in INS-1 Cells. Molecules 17, 10052-10064. doi:10.3390/molecules170910052

Wang, Z. (2017). ErbB Receptors and Cancer. Methods Mol. Biol. 1652, 3-35. doi:10.1007/978-1-4939-7219-7_1

Wu, X.-F., Xu, R., Ouyang, Z.-J., Qian, C., Shen, Y., Wu, X.-D., et al. (2013). Beauvericin Ameliorates Experimental Colitis by Inhibiting Activated T Cells via Downregulation of the PI3K/Akt Signaling Pathway. PLoS One 8, e83013, doi:10.1371/journal.pone.0083013

Xu, B.-L., Zhang, G.-J., and Ji, Y.-B. (2015). Active Components Alignment of Gegenqinlian Decoction Protects Ulcerative Colitis by Attenuating Inflammatory and Oxidative Stress. J. Ethnopharmacology 162, 253-260. doi:10.1016/j.jep.2014.12.042

Xu, X., Zhang, W., Huang, C., Li, Y., Yu, H., Wang, Y., et al. (2012). A Novel Chemometric Method for the Prediction of Human Oral Bioavailability. Int. J. Mol. Sci. 13, 6964-6982. doi:10.3390/ijms13066964

Yan, M. W., Mei, Q. K., Yan, B. C., Zhi, X. X., and Rui, F. W. (2012). Clinical Study of Internal and External Treatment of Gegen Qinlian Wutan Decoction in Active Ulcerative Colitis. Chin. J. Exp. Traditional Med. Formulae 18, 267-270. doi:10.13422/j.cnki.syfjx.2012.17.005

Yan, Y.-x., Shao, M.-j., Qi, Q., Xu, Y.-s., Yang, X.-q., Zhu, F.-h., et al. (2018b). Artemisinin Analogue SM934 Ameliorates DSS-Induced Mouse Ulcerative Colitis via Suppressing Neutrophils and Macrophages. Acta Pharmacol. Sin 39, 1633-1644. doi:10.1038/aps.2017.185

Yan, Y., Du, C., Li, Z., Zhang, M., Li, J., Jia, J., et al. (2018a). Comparing the Antidiabetic Effects and Chemical Profiles of Raw and Fermented Chinese GeGen-Qin-Lian Decoction by Integrating Untargeted Metabolomics and Targeted Analysis. Chin. Med. 13, 54. doi:10.1186/s13020-018-0208-7

Yang, G.-Y., Taboada, S., and Liao, J. (2009). Inflammatory Bowel Disease: a Model of Chronic Inflammation-Induced Cancer. Methods Mol. Biol. 511, 193-233. doi:10.1007/978-1-59745-447-6_9

Yang, W., Zhang, Y., Wu, W., Huang, L., Guo, D., and Liu, C. (2017). Approaches to Establish Q-Markers for the Quality Standards of Traditional Chinese Medicines. Acta Pharmaceutica Sinica B 7, 439-446. doi:10.1016/j.apsb.2017.04.012

Yao, D., Dong, M., Dai, C., and Wu, S. (2019). Inflammation and Inflammatory Cytokine Contribute to the Initiation and Development of Ulcerative Colitis and its Associated Cancer. Inflamm. Bowel Dis. 25, 1595-1602. doi:10.1093/ibd/izz149

Yarden, Y., and Pines, G. (2012). The ERBB Network: at Last, Cancer Therapy Meets Systems Biology. Nat. Rev. Cancer 12, 553-563. doi:10.1038/ nrc3309

Yu, L. Z., Wu, J. Y., Luo, J. B., Huang, X. G., Lin, H., and Xing, Y. L. (2005). Experimental Study on Anti-diarrhae Effect of Gegenqinlian Decoction and its Compounds. Chin. Traditional Patent Med. 27, 300-304.

Zhang, L.-C., Wang, Y., Tong, L.-C., Sun, S., Liu, W.-Y., Zhang, S., et al. (2017). Berberine Alleviates Dextran Sodium Sulfate-Induced Colitis by Improving Intestinal Barrier Function and Reducing Inflammation and Oxidative Stress. Exp. Ther. Med. 13, 3374-3382. doi:10.3892/etm.2017.4402

Zhu, L., Gu, P., and Shen, H. (2019). Protective Effects of Berberine Hydrochloride on DSS-Induced Ulcerative Colitis in Rats. Int. Immunopharmacology 68, 242-251. doi:10.1016/j.intimp.2018.12.036

Conflict of Interest: The authors declare that the research was conducted in the absence of any commercial or financial relationships that could be construed as a potential conflict of interest.

Copyright (c) $2021 \mathrm{Liu}, \mathrm{Fan}, \mathrm{Du}, \mathrm{Mei}$ and Fu. This is an open-access article distributed under the terms of the Creative Commons Attribution License (CC $B Y)$. The use, distribution or reproduction in other forums is permitted, provided the original author(s) and the copyright owner(s) are credited and that the original publication in this journal is cited, in accordance with accepted academic practice. No use, distribution or reproduction is permitted which does not comply with these terms. 